\title{
Pressure Transient Solutions for Vertically Slotted-Partially Penetrated Vertical Wells in Porous Media
}

\author{
Denis Biryukov • Fikri J. Kuchuk
}

Received: 6 December 2013 / Accepted: 22 October 2014 / Published online: 8 November 2014

(C) The Author(s) 2014. This article is published with open access at Springerlink.com

\begin{abstract}
In this paper, we present semi-analytical pressure transient solutions for vertically slotted-limited entry or partially penetrated vertical wells. These solutions are needed for interpretation of the pressure transient tests conducted with the Wireline Formation Testing vertically slotted-packer configuration, and in wells with vertically slotted-liner completions. These slots have limited size openings and are vertically placed on a non-permeable cylindrical wellbore in a porous medium. The fluid from the formation is produced through these slots into the wellbore. Pressure transient solutions are not readily available for the uniform pressure boundary condition on the surface of the slots because such a condition creates a mixed boundary value problem, which is difficult to solve. Here we present exact pressure transient solutions obtained under the assumption that the pressure on the slot surface is uniform but a priori unknown, and the rest of the wellbore surface is non-permeable (no-flow condition). Furthermore, we generalize our solution for the case of multiple slots (open sections) on the wellbore for both well testing and Wireline formation tester packer modules. Our solutions are compared with the existing solutions. A new formula for obtaining the drawdown horizontal mobility is presented for anisotropic media.
\end{abstract}

Keywords Pressure transient solutions - Slotted-partially penetrated vertical wells . Pressure transient testing $\cdot$ Slotted dual-packer module $\cdot$ Mobility estimation

\section{List of Symbols}

$\begin{array}{ll}C & \text { Wellbore storage constant, } \mathrm{RB} / \mathrm{psi}\left(\mathrm{m}^{3} / \mathrm{Pa}\right) \\ c & \text { Compressibility, } \mathrm{psi}^{-1}\left(\mathrm{~Pa}^{-1}\right) \\ \text { se \& ce } & \text { Normalized angular even and odd Mathieu functions } \\ \text { erf } & \text { Error function } \\ h & \text { Formation thickness, } \mathrm{ft}[\mathrm{m}]\end{array}$

D. Biryukov · F. J. Kuchuk $(\bowtie)$

1 rue Henri Becquerel, 92142 Clamart Cedex, France

e-mail: kuchuk1@slb.com 


$\begin{array}{ll}g & \text { Unit impulse response } \\ \mathrm{I} & \text { Modified Bessel function of the first kind } \\ \mathrm{J} & \text { Bessel function of the first kind } \\ \mathrm{K} & \text { Modified Bessel function of the second kind } \\ k & \text { Permeability, md }\left(\mathrm{m}^{2}\right) \\ l & \text { Characteristic length or Half-length, } \mathrm{ft}(\mathrm{m}) \\ \mathcal{L} & \text { Laplace transform } \\ \mathcal{L}^{-1} & \text { Inverse Laplace transform } \\ \mathrm{Mc} \& \mathrm{Ms} & \text { Radial even and odd Mathieu functions of the third kind } \\ p & \text { Pressure, psi (Pa) } \\ q & \text { flow rate, } \mathrm{RB} / \mathrm{D}\left(\mathrm{m}^{3} / \mathrm{s}\right) \\ r & \text { Radius or radial coordinate } \\ s & \text { Laplace transform variable } \\ T & \text { Chebyshev polynomial } \\ t & \text { Time, h (s) } \\ x & \text { Coordinate } \\ y & \text { Coordinate } \\ z & \text { Vertical coordinate } \\ \alpha & \text { Constant } \\ \beta & \text { Constant } \\ \gamma & \text { Constant } \\ \delta & \text { Constant } \\ \eta & \text { Diffusivity for pressure } \\ \mu & \text { Viscosity, cp (Pa s) } \\ \phi & \text { Porosity, fraction } \\ \tau & \text { Dummy variable } \\ \xi & \text { Dummy variable } \\ & \end{array}$

\section{Subscripts}

$\begin{array}{ll}a & \text { Pressure-averaged } \\ D & \text { Dimensionless } \\ f & \text { Formation } \\ h & \text { Horizontal } \\ m & \text { Measured } \\ o & \text { Initial or original } \\ s f & \text { Sandface } \\ u & \text { Uniform pressure } \\ v & \text { Vertical } \\ w & \text { Wellbore }\end{array}$

\section{Introduction}

Pressure transient tests are conducted along the wellbore to obtain reservoir pressure and a discrete permeability (horizontal and vertical permeabilities) distribution with the wireline formation testers (WFT), shown in Fig. 1 (Kuchuk et al. 2010; Zimmerman et al. 1990). A wireline formation tester consists of a number of modules and allows an unlimited number of 


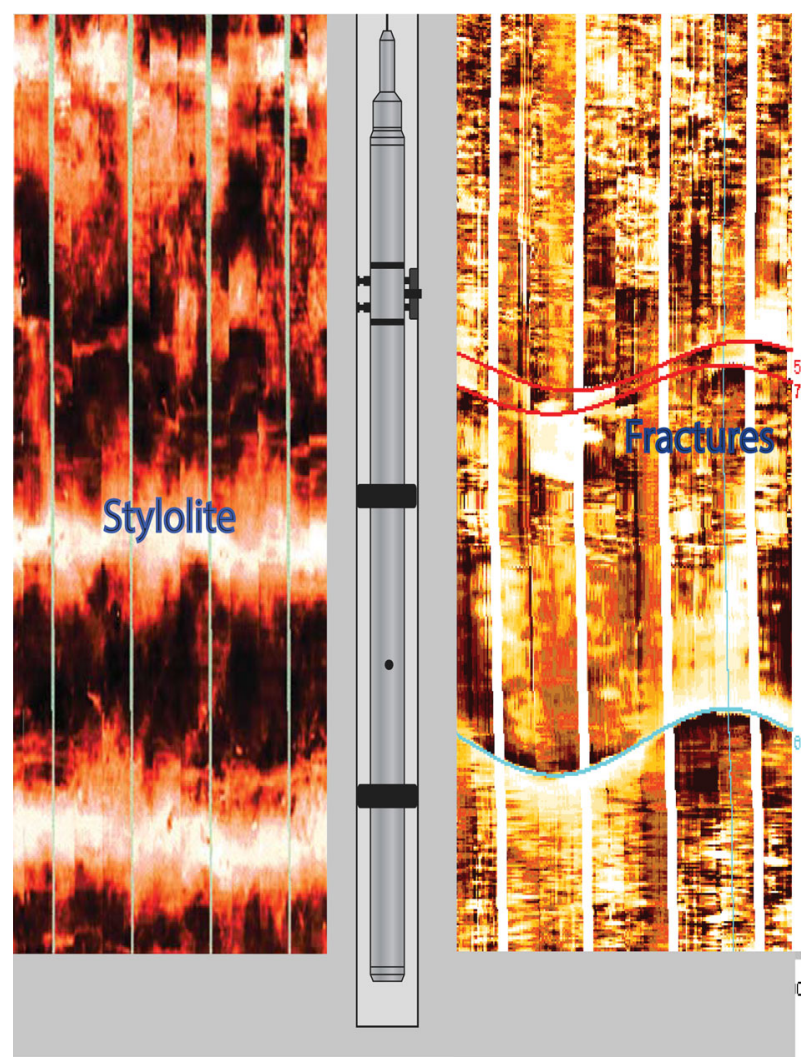

Fig. 1 WFT Packer-Probe tool combination with the formation resistivity images along the wellbore: the FMI image (left) shows low-porosity stylolite streaks (white) separated and the FMI image (right) shows a multiple-fractured zone and a faulted zone at the bottom

formation pressure measurements, formation testing, and multiple formation fluid sampling. The tool is capable of conducting interval pressure transient tests (IPTT) using the wireline conveyance systems.

Wireline Formation Testing (WFT) (Zimmerman et al. 1990; Kuchuk et al. 2010) tools are nowadays provided by several companies, and come with widely varying characteristics in terms of size, weight, pressure and temperature ratings, power requirements, measurement devices specifications, pumping power, etc. As we stated above, WFT tools offer these basic functions along the wellbore: (1) measure formation pressure via short pretests, (2) perform pressure transient tests, (3) perform single and multipoint interval (interference) tests, (4) make flow rate measurements, (5) take formation fluid samples and provide basic downhole fluid analysis, and (6) conduct stress tests. To perform all these functions, WFT tools have many modules that provide hydraulic and electrical powers, pressure gauge carries, sampling bottles and chambers, downhole fluid analyzer, and pumps for production/injection from the formation into the wellbore or visa versa. The conventional small-area sink probe (also sometimes referred to as the standard single probe) has been the main WFT producing and testing unit from the beginning, and has expanded and modified (dual-packer, guard probe, etc.) over the years. 
For the purpose of pretests and pressure transient testing, the most important components of a WFT string are the probes or dual-packers (Fig. 1) through which the flow from the formation into the tool string takes place, and where both pressure and flow rate are recorded. Different types of probes and dual-packers exist and can be assembled in different ways. Beyond these essential elements, other modules also are necessary to perform the flow operations, such as pumps, power modules, sample chambers, etc. The packer module has two packer elements that are inflated to isolate about $1 \mathrm{~m}(3.3 \mathrm{ft})$ wellbore interval, as shown in Fig. 1. Because a large section of the sandface is open to flow between two packer elements, the production interval of the formation is several thousand times larger than that of conventional probes. Unlike the flow geometry of conventional probes, the radial symmetry about the wellbore axis is maintained with the dual-packer geometry during the flow in laterally anisotropic homogenous reservoirs, and is slightly distorted in vertically anisotropic systems. Therefore, the dual-packer module is very useful for conducting pressure transient tests in heterogeneous, layered, shaly, fractured, and vuggy formations. In these type of formations, it is not difficult to sustain the hydraulic isolation of the tested section from the rest of the wellbore, particularly in fractured and vuggy formations. As shown in Fig. 1, the dual-packer envelops a matrix, fracture and/or fault zone and is set against the borehole wall to hydraulically isolate the tested section, see for instance, Zeybek et al. (2002) for the details of packer-probe transient tests that are conducted across the fracture zones.

The dual-packer module is also very useful for WFT testing of unconsolidated formations and condensate reservoirs because the packer production area of the sandface several thousand times larger than the production area of conventional probes (Kuchuk et al. 2010). In other words, the wellbore pressure drop is very small during transient tests and sampling with the parker module compared to that of conventional probes even though production rate could be usually much higher for the parker module. It is well known that the sand production into the wellbore is extremely sensitive to the pressure drop during WFT testing and sampling of unconsolidated formations. Condensate reservoirs are also extremely sensitive to the pressure change. If the wellbore pressure drops below the dewpoint during testing and sampling, liquid condenses from gas and form a condensate bank, which highly complicates both testing and sampling.

In some respects, a dual-packer pressure transient test resembles a small scale DST-type test, although the radius of investigation of a dual-packer pressure transient test is not more than a few tens of feet $(10-50 \mathrm{ft})$. A longer production time will increase the radius of investigation very little because a spherical flow is established in the vicinity of the wellbore due to a small-length production interval, unless the formation is thin. The dual-packer module with one or two probes is also very useful for permeability profiling along the wellbore, particularly in layered reservoirs. For instance, as shown in Fig. 1, the FMI image (left) shows three low-porosity stylolite streaks (white) separated by dark very permeable intervals. The top stylolite streak is particularly patchy. Usually, the stylolite intervals may be thinner than $1 \mathrm{ft}$, therefore, conventional probes are not suitable to test these stylolite zones. The same figure also shows the FMI image (right), where a multiple-fractured zone and a faulted zone at the bottom can be observed. Again conventional probes are not suitable to test these fractured and faulted zones.

The recently introduced vertically slotted-packer probe (Saturn ${ }^{1} 3 \mathrm{D}$ radial packer module), as shown in Fig. 2, provides a new type of 4-Dimensional pressure transient test data. As shown in Fig. 3, the packer has four rectangular slots with a height of $188 \mathrm{~mm}(0.6168 \mathrm{ft})$ and a width of $74.5 \mathrm{~mm}(0.2444 \mathrm{ft})$. The tips of each slot are slightly oval-shaped. The four

1 Mark of Schlumberger. 
Fig. 2 Schematic of the vertically slotted-packer module: a Vertical flow position of probes. b Flow distribution in the formation through four slots, after Wireline-Schlumberger (2012)

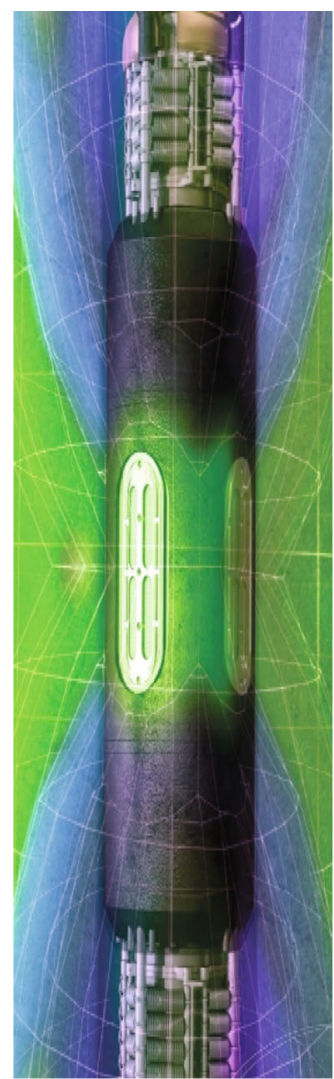

(a)

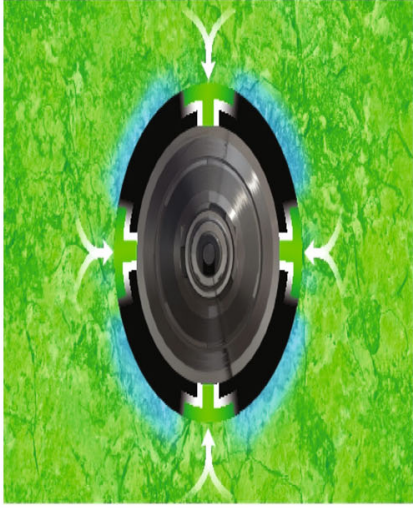

(b)

slot packer module is circumferentially equally spaced over the cylindrical surface of the packer element (see Fig. 2). In addition to 4-D pressure transient tests, the slotted-packer module allows us to obtain reliable pressure profiles and fluid samples in difficult reservoir and well conditions, such as low-permeability and unconsolidated formations, heavy oil and near-critical fluids, and rugose boreholes (Wireline-Schlumberger 2012).

Recently Al-Otaibi et al. (2012) and Flores de Dios et al. (2012) presented two papers on the applications of the slotted-packer module. The first paper dealt with downhole fluid sampling and transient tests in low mobility carbonate formations. They used a commercial numerical simulator and an analytical model (Kuchuk 1994; Wilkinson and Hammond 1990), which is a transient solution for a partially penetrated well to interpret tests. Of course, this solution does not accurately model the early time behavior of the slotted-packer module. The Flores de Dios et al. (2012) paper mainly dealt with taking downhole fluid samples using the slotted packer module in a heavy-oil unconsolidated-sandstone reservoir $\left(\approx 8^{\circ} \mathrm{API}\right)$.

The testing, solutions, and interpretation of $2 \mathrm{D}$ and $3 \mathrm{D}$ interval pressure transient tests (IPTT) with the dual-packer module and with one- or two-probe combination (Fig. 1) were given by Pop et al. (1993), Kuchuk (1994, 1998), Kuchuk and Onur (2003), Onur et al. (2004a,b), Onur and Kuchuk (1999), Kuchuk et al. (2010). Solutions and interpretation models are not available for the 4D IPTTs conducted with the vertically slotted-packer tool, as shown in Fig. 2. With appropriate solutions, slotted-packer 4D IPTTs may provide an estimation of permeabilities in the $x, y$, and $z$ directions. 
Fig. 3 Schematic of the vertically slotted-packer module with its dimensions, where the origin the coordinate system is on the well axis at

$\left\{x=r_{w}, y=0, z=0\right\}$ which corresponds to the center of the probe

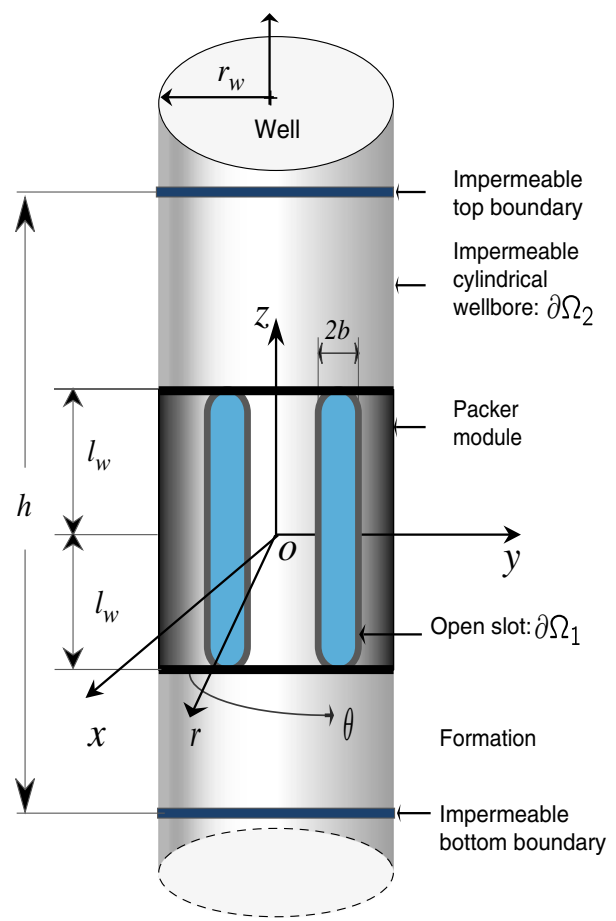

The earlier solutions for the pressure transient behavior of partially penetrated wells (Odeh 1968; Hantush 1957; Nisle 1958; Brons and Marting 1961) used the line source approximation to derive the finite-length uniform-flux line source solution, from which the wellbore pressure was approximated at the middle point of the open section of the well. The second set of solutions is based on either finite uniform-flux line source approximations with an appropriate equivalent-pressure point (Gringarten and Ramey 1975) or uniform-flux pressure-averaged approximations (Streltsova-Adams 1979; Yildiz and Bassiouni 1990; Kuchuk and Wilkinson 1991; Kuchuk 1994; Wilkinson and Hammond 1990; Ozkan and Raghavan 1991; Biryukov and Kuchuk 2012). The third set of solutions is based on the uniform-pressure boundary condition, whioch is the true wellbore condition, on the surface of the open interval given by Papatzacos $(1987 \mathrm{a}, \mathrm{b})$, in which they used the prolate spherical coordinates to solve the pressure diffusion equation for partially penetrated wells.. Although it is an exact infiniteconductivity solution, the partially penetration geometry can only be mapped approximately to a prolate spheroid.

Recently Biryukov and Kuchuk (2012) presented exact pressure transient solutions obtained under the assumption that the pressure on the open surface is uniform but a priori unknown, and the rest of the wellbore surface is non-permeable (no-flow condition). Furthermore, they generalize their solution for the case of multiple production sections on the wellbore for both well testing and wireline formation tester packer modules. They compared their solutions with the middle point, equivalent-pressure point, and pressure-averaging solutions given in the petroleum literature. In addition to the recent Biryukov and Kuchuk (2012) paper, a few other papers were also published on selectively perforated and/or completed wells, i.e., wells with multiple production slots (ring slots along the wellbore), specially Spivak and Horne (1983), Larsen (1993), Yildiz and Cinar (1998), Tang et al. (2000), Yildiz (2002a, b, 2004, 2006a, b). The Spivak and Horne (1983) vertically slotted-liner solution, 
which is obtained from the uniform-flux solution, is somewhat similar to the solutions given in this paper. However, there are some fundamental differences, such as where to evaluate the pressure over the sandface, because it changes for these types of slot-line solutions when the effects of the bottom and top boundary are felt in the wellbore. Furthermore, we consider anisotropic media, while the Spivak and Horne (1983) solution is for isotropic systems. They considered both ring and vertical slots along the wellbore.

In this paper, we develop "exact" solutions to boundary value problems for the slottedpacker module with uniform pressure and uniform-flux inner boundary conditions on the cylindrical surface of a well in a three-dimensional porous medium internally bounded by an impermeable cylindrical wellbore. These solutions are also extended to formations that are bounded by no-flow top and bottom boundaries.

\section{Mathematical Model}

Let us consider pressure diffusion of a single-phase constant viscosity and slightly compressible fluid in a three-dimensional infinite anisotropic non-deformable homogeneous porous medium bounded by $-\infty<x<\infty,-\infty<y<\infty$, and $-\infty<z<\infty$, in which there are no sinks and/or sources. The permeabilities of the media in the principle directions are denoted by $k_{x}, k_{y}$, and $k_{z}$. It is assumed that fluid viscosity $\mu$, porosity $\phi$, and total compressibility $c_{t}$ are pressure- and time-invariant and uniform throughout the reservoir. In this paper, we assume that the principle axes of the permeability tensor coincide with the principle axes of the coordinate system we use. If this is not the case, for a given formation, we can always find a new coordinate system whose axes coincide with the permeability tensor. However, for deviated wells in formations with horizontal layers, or vertical, deviated, or horizontal wells in tilted formations, the coordinate transformation is complicated (Besson 1990; Onur et al. 2004a, 2011).

Single-phase pressure diffusion in an anisotropic homogeneous porous medium (shown in Fig. 3), defined above, can be described by the following partial differential equation (Muskat 1937)

$$
\lambda_{x} \frac{\partial^{2} \mathcal{P}}{\partial x^{2}}+\lambda_{y} \frac{\partial^{2} \mathcal{P}}{\partial y^{2}}+\lambda_{z} \frac{\partial^{2} \mathcal{P}}{\partial z^{2}}=\varphi \frac{\partial \mathcal{P}}{\partial t},-\infty<x, y, z<\infty, t>0,
$$

subject to the initial condition

$$
\mathcal{P}(\mathbf{x}, t)=h(\mathbf{x}) \quad-\infty<x, y, z<\infty, \quad t=0,
$$

and the outer boundary condition

$$
\mathcal{P}(\mathbf{x}, t)=0, x, y, z \rightarrow \pm \infty, t>0,
$$

where the spatial position vector is $\mathbf{x}=x \mathbf{i}+y \mathbf{j}+z \mathbf{k}$ and $r=|\mathbf{x}|=\sqrt{x^{2}+y^{2}+z^{2}}, \mathbf{i}, \mathbf{j}$, and $\mathbf{k}$ are the unit vectors in the $x, y$, and $z$ directions, respectively. $\mathcal{P}(\mathbf{x}, t)=p_{0}(\mathbf{x}, 0)-p(\mathbf{x}, t)$, $h(\mathbf{x})=p_{0}-p(\mathbf{x}, 0), p(\mathbf{x}, 0)$ is the initial pressure distribution imposed at $t \leq 0$. For most of our problems, without a loss of generality, $p_{0}(\mathbf{x}, 0) \equiv p_{0}$, thus $h(\mathbf{x})=0$, where $p_{0}$ is the initial reservoir pressure. For the MBVPs considered here, we also assume the medium to be transversely anisotropic $\left(k_{x}=k_{y}=k_{h}\right.$ and $\left.k_{z}=k_{v}\right)$, i.e., the permeability varies very little directionally in the horizontal plane, which is reasonable for spatial scales of well testing and wireline formation testers.

The inner boundary conditions of Eq. 1 for the pressure transient problems we considered are of the mixed type. In a mixed boundary value problem (MBVP), we have both the 
Neumann and Dirichlet boundary conditions imposed on the inner boundary surface. For instance, as shown in Fig. 3, the boundary condition at the open (producing) section of the inner boundary $\partial \Omega_{1}$, for which the total volumetric flow rate $q$ is specified, can be written as

$$
-\iint_{\partial \Omega_{1}}\{\boldsymbol{n} \cdot \boldsymbol{T} \operatorname{grad}[\mathcal{P}(\mathbf{x}, t)]\} \mathrm{d} S=q(t) \quad \text { on } \partial \Omega_{1}, \quad t>0,
$$

where $\boldsymbol{n}$ is the outer normal vector to the surface $\partial \Omega_{1}, \boldsymbol{T}$ is the mobility tensor, $q$ is the total flux outwards from the surface $\partial \Omega_{1}$, and for the no-flow section of the inner boundary

$$
\{\boldsymbol{n} \cdot \boldsymbol{T} \operatorname{grad}[\mathcal{P}(\mathbf{x}, t)]\}=0 \quad \text { on } \partial \Omega_{2}, \quad t>0 .
$$

Furthermore, we also require the pressure (potential) at the open surface $\partial \Omega_{1}$ to be uniform (independent of $\mathbf{x}$ ), which implies that

$$
\mathcal{P}(\mathbf{x}, t)=\mathcal{P}_{w u}(t) \quad \text { on } \partial \Omega_{1}, \quad t>0 .
$$

The inner boundary conditions specified by Eqs. 4-6 over the surfaces $\partial \Omega_{1}$ and $\partial \Omega_{2}$ create a mixed boundary value problem.

In this paper, we will present solutions for the constant sandface flow rate $\left(q_{s f}\right)$. If the flow rate varies, then wellbore pressure at any time can be written from the convolution integral (Muskat 1937), which is Duhamel's superposition principle, as

$$
p_{w}(t)=p_{o}-\int_{0}^{t} q_{s f}(\tau) g_{w}(t-\tau) \mathrm{d} \tau
$$

and in the Laplace domain

$$
\bar{p}_{w}(s)=\frac{p_{o}}{s}-\bar{q}_{s f}(s) \bar{g}_{w}(s),
$$

where $t$ is time and $g_{w}$ is the impulse response at the wellbore. If the flow rate is constant at the surface, the wellbore pressure, including skin and storage effects in the Laplace domain, can be written from Eq. 8 (see pages 60-66 of Kuchuk et al. (2010) for the details) as

$$
\bar{p}_{w}(s)=\frac{p_{o}}{s}-\frac{q g_{w}(s)}{s\left[1+C s \bar{g}_{w}(s)\right]} .
$$

Equation 9 in terms of the dimensionless wellbore impulse response can be written as

$$
\bar{p}_{w D}(s)=\frac{\bar{g}_{w D}(s)}{1+C_{D} s \bar{g}_{w D}(s)},
$$

and in terms of the dimensionless pressure and skin $(S)$ as

$$
\bar{p}_{w D}\left(s_{D}\right)=\frac{s \bar{p}_{D}(s)+S}{s\left\{1+C_{D} s\left[s \bar{p}_{D}(s)+S\right]\right\}},
$$

where

$$
p_{w D}=\frac{4 \pi k_{h} l_{w} \Delta p_{w}}{q \mu}, \quad t_{D}=\frac{k_{h} t}{\phi \mu c_{t} r_{w}^{2}}, \quad C_{D}=\frac{C}{2 \pi \phi c_{t} h r_{w}^{2}},
$$

$C$ is the wellbore storage coefficient, $S$ is the skin factor, and $s$ is the Laplace transform variable that is related to the dimensionless time $t_{D}$. The above equations in this section provide a general framework for interpreting simultaneously measured pressure data with an arbitrarily varying flow rate as a function of time for both pressure transient well and wireline formation testing. The dimensionless $p_{D} \mathrm{~s}$ for the formation pressure with the inner boundary 
conditions of Eq. 1 will be presented in the following sections. If the flow rate is variable at the sandface or surface, the convolution integral given by Eq. 7 and its Laplace transform given by Eqs. 8 should be used to obtain the wellbore pressure. Therefore, to simplify the below derivations, we will assume everywhere that total flow rate $q(t) \equiv q$ is independent of time.

\section{Pressure Transient Solutions for Vertical Multiple Slots (Openings) on a Partially Penetrated Well}

In the following subsection, we first obtain solutions for a vertical single slot on a partially penetrated well in an unbounded porous medium. Then the solutions will be generalized for both vertically bounded systems and multiple slots, as shown Fig. 3.

\subsection{Single-Slot Solution}

Consider one of the vertical slots, as shown in Fig. 3, with a thickness (height) of $2 l_{w}$ and a width of $2 b$ on a partially penetrated well in an unbounded porous medium. The flow rate $q$ through the slot from the formation into the wellbore is assumed to be constant. The slot surface is equipotential, i.e., is under uniform but a priori unknown pressure change $\mathcal{P}_{w u}(t)=p_{0}(t)-p_{w u}(t)$, where subscripts $w$ and $u$ denote wellbore and uniform pressure, respectively. The reservoir is assumed to be infinite in all directions (solutions for the reservoir bounded in the vertical direction $z$ will be presented in the next section), with porosity $\phi$ and permeability equal to $k_{h}$ in horizontal and $k_{v}$ in vertical directions. The fluid viscosity $\mu$ and total compressibility $c_{t}$ are constant. Using the mathematical model described above, the single-slot partially penetrated well pressure transient problem can be formulated as

$$
\begin{aligned}
& \lambda_{h} \frac{1}{r} \frac{\partial}{\partial r} r \frac{\partial \mathcal{P}}{\partial r}+\lambda_{h} \frac{1}{r^{2}} \frac{\partial^{2} \mathcal{P}}{\partial \theta^{2}}+\lambda_{v} \frac{\partial^{2} \mathcal{P}}{\partial z^{2}}=\varphi \frac{\partial \mathcal{P}}{\partial t}, \\
& \mathcal{P}(r, \theta, z, 0)=0, \\
& \mathcal{P}\left(r_{w}, z, \theta, t\right)=\mathcal{P}_{w u}(t), \text { for }|z|<l_{w} \text { and }\left|r_{w} \theta\right|<b, \\
& \frac{\partial \mathcal{P}}{\partial r}\left(r_{w}, \theta, z, t\right)=0, \text { for }|z| \geq l_{w} \text { or }\left|r_{w} \theta\right| \geq b, \\
& \mathcal{P}(r, z, \theta, t) \rightarrow 0 \text { as } \sqrt{r^{2}+z^{2}} \rightarrow \infty, \\
& r_{w} \lambda_{h} \int_{-b / r_{w}}^{b / r_{w}} \int_{-l_{w}}^{l_{w}} \frac{\partial \mathcal{P}}{\partial r}\left(r_{w}, \theta, z, t\right) \mathrm{d} z \mathrm{~d} \theta=-q,
\end{aligned}
$$

where mobilities are defined as $\lambda_{h}=\frac{k_{h}}{\mu}$ and $\lambda_{v}=\frac{k_{v}}{\mu}$, and $\varphi=\phi c_{t}$. Due to symmetry about the horizontal and vertical planes, we will further treat the problem only in the domain $\{\theta \geq 0, z \geq 0\}$. Let us define the following dimensionless variables as

$$
p_{D}=\frac{4 k_{h} \pi l_{w} \mathcal{P}}{q \mu} ; \quad p_{D w u}=\frac{4 k_{h} \pi l_{w}}{q \mu} \mathcal{P}_{w u} ; \quad b_{D}=\frac{b}{r_{w}} ; \quad r_{D}=\frac{r}{r_{w}} ; \quad z_{D}=\frac{z}{l_{w}} ; \quad v^{2}=\frac{k_{v}}{k_{h}} \frac{r_{w}^{2}}{l_{w}^{2}},
$$

and $t_{D}$ is defined in Eq. 12. Then the mathematical model given above can be written in dimensionless form as

$$
\frac{1}{r_{D}} \frac{\partial}{\partial r_{D}} r_{D} \frac{\partial p_{D}}{\partial r_{D}}+\frac{1}{r_{D}^{2}} \frac{\partial^{2} \mathcal{P}}{\partial \theta^{2}}+v^{2} \frac{\partial^{2} p_{D}}{\partial z_{D}^{2}}=\frac{\partial p_{D}}{\partial t_{D}}
$$




$$
\begin{aligned}
& p_{D}\left(r_{D}, \theta, z_{D}, 0\right)=0, \\
& p_{D}\left(1, \theta, z_{D}, t_{D}\right)=p_{D w u}\left(t_{D}\right), \text { for } 0 \leq z_{D}<1 \text { and } 0 \leq \theta<b_{D}, \\
& \frac{\partial p_{D}}{\partial r_{D}}\left(1, \theta, z_{D}, t_{D}\right)=0, \text { for } z_{D} \geq 1 \text { or } \theta \geq b_{D}, \\
& p_{D}\left(r_{D}, \theta, z_{D}, t_{D}\right) \rightarrow 0 \text { as } \sqrt{r_{D}^{2}+z_{D}^{2}} \rightarrow \infty, \\
& \frac{1}{\pi} \int_{0}^{b_{D}} \int_{0}^{1} \frac{\partial p_{D}}{\partial r_{D}}\left(1, \theta, z_{D}, t_{D}\right) \mathrm{d} z_{D}=-1 .
\end{aligned}
$$

Equations 22 and 25 can be further rewritten in the following form

$$
\frac{\partial p_{D}}{\partial r_{D}}\left(1, \theta, z_{D}, t_{D}\right)=-q_{D}\left(\theta, z_{D}, t_{D}\right), \text { for } 0 \leq z_{D}<1 \text { and } 0 \leq \theta<b_{D},
$$

where the function $q_{D}\left(\theta, z_{D}, t_{D}\right)$ is a priori unknown and will be specified later. Using the boundary conditions given by Eqs. 23 and 26, the solution to Eq. 20 can be written as

$$
p_{D}\left(r_{D}, \theta, z_{D}, t_{D}\right)=\int_{0}^{t_{D}} \int_{0}^{1} \int_{0}^{b_{D}} q_{D}(\psi, u, \tau) \mathrm{G}\left(r_{D}, \theta, \psi, z_{D}, u, t_{D}-\tau\right) \mathrm{d} u \mathrm{~d} \psi \mathrm{d} \tau,
$$

where $\mathrm{G}$ is a Green's function for the corresponding problem which can be expressed as

$$
\mathrm{G}\left(r_{D}, \theta, \psi, z_{D}, u, t_{D}\right)=\Theta\left(r_{D}, \theta, \psi, t_{D}\right) Z\left(z_{D}, u, t_{D}\right)
$$

with

$$
\begin{aligned}
& \Theta\left(r_{D}, \theta, \psi, t_{D}\right)=\mathcal{L}^{-1}\left\{\frac{1}{\sqrt{s}} \sum_{n=0}^{\infty}\left(1-\frac{\delta_{0 n}}{2}\right) \frac{2 \mathrm{~K}_{n}\left(r_{D} \sqrt{s}\right) \cos (n \theta) \cos (n \psi)}{\mathrm{K}_{n+1}(\sqrt{s})+\mathrm{K}_{n-1}(\sqrt{s})}\right\}, \\
& \mathrm{Z}\left(z_{D}, u, t_{D}\right)=\frac{2 e^{-\left(z_{D}-u\right)^{2} /\left(4 v^{2} t_{D}\right)}}{\sqrt{\pi t_{D}} v} .
\end{aligned}
$$

where

$$
\delta_{i j}=\left\{\begin{array}{l}
1 \text { when } i=j, \\
0 \text { when } i \neq j
\end{array}\right.
$$

Now let us assume that the slot flux density $q_{D}(\theta, u, \tau)$ can be expressed as

$$
q_{D}(\theta, u, \tau)=\frac{1}{\pi} \sum_{i=0}^{\infty} \sum_{j=0}^{\infty} c_{i j}(t) \frac{(-1)^{i} T_{2 i}\left(\theta / b_{D}\right)}{\sqrt{b_{D}^{2}-\theta^{2}}} \frac{T_{2 j}\left(z_{D}\right)}{\sqrt{1-z_{D}^{2}}}
$$

where $T$ is the Chebychev polynomial and the time functions $c_{i j}$ will be determined later. The factor $\frac{1}{\sqrt{b_{D}^{2}-\theta^{2}} \sqrt{1-z_{D}^{2}}}$ reflects the main singularity of the flux density near the slot tips - its divergence - and insures fast convergence of the series.

Substituting $q_{D}$ in this form into Eq. 27, we can obtain the following expression for the dimensionless reservoir pressure:

$$
p_{D}\left(r_{D}, \theta, z_{D}, t_{D}\right)=\sum_{i=0}^{\infty} \sum_{j=0}^{\infty} \int_{0}^{t_{D}} c_{i j}(\tau) \Theta_{2 i}\left(r_{D}, \theta, t_{D}-\tau\right) \mathrm{Z}_{2 j}\left(z_{D}, t_{D}-\tau\right) \mathrm{d} \tau,
$$


where

$$
\begin{aligned}
& \Theta_{2 i}\left(r_{D}, \theta, t_{D}\right)=\mathcal{L}^{-1}\left\{\frac{1}{\sqrt{s}} \sum_{n=0}^{\infty}\left(1-\frac{\delta_{0 n}}{2}\right) \frac{2 \mathrm{~K}_{n}\left(r_{D} \sqrt{s}\right) \mathrm{J}_{2 i}\left(n b_{D}\right) \cos (n \theta)}{\mathrm{K}_{n+1}(\sqrt{s})+\mathrm{K}_{n-1}(\sqrt{s})}\right\}, \\
& \mathrm{Z}_{2 j}\left(z_{D}, u, t_{D}\right)=\frac{1}{\sqrt{\pi t_{D}} v} \int_{0}^{\pi} e^{-\left(z_{D}-\cos \eta\right)^{2} /\left(4 v^{2} t_{D}\right)} \cos (2 j \eta) \mathrm{d} \eta .
\end{aligned}
$$

Although these functions seem complicated, we will show in Appendix 1 how they can be easily evaluated. It should be observed that the function $p_{D}\left(r_{D}, \theta, z_{D}, t_{D}\right)$ satisfies the boundary condition defined by Eq. 23. The unknown functions $c_{i j}\left(t_{D}\right)$ and the uniform wellbore pressure $p_{D w u}\left(t_{D}\right)$ can be obtained from the boundary conditions specified by Eqs. 22 and 25, implying that

$$
\begin{gathered}
\sum_{i=0}^{\infty} \sum_{j=0}^{\infty} \int_{0}^{t_{D}} c_{i j}(\tau) \Theta_{2 i}\left(1, \theta, t_{D}-\tau\right) \mathrm{Z}_{2 j}\left(z_{D}, t_{D}-\tau\right) \mathrm{d} \tau=p_{D w u}\left(t_{D}\right) \\
\text { for } 0 \leq z_{D}<1 \text { and } 0 \leq \theta<b_{D}, \\
\qquad c_{00}\left(t_{D}\right)=1 .
\end{gathered}
$$

To solve this system of integral equations efficiently, we must use a finite number of unknown functions $c_{i j}\left(t_{D}\right)$, and discretize Eq. 37 on a set of collocation points. Due to the nature of the basis functions we used to expand $q_{D}\left(\theta, z_{D}, t_{D}\right)$, we suggest to take a set of Chebyshev nodes $z_{D m}=\cos \left[\frac{\pi}{2 M}(0.5+m)\right], m=0, \ldots, M-1$ and $\theta_{k}=b_{D w} \cos \left[\frac{\pi}{2 K}(0.5+k)\right], k=$ $0, \ldots, K-1$. Furthermore, we also discretize Eq. 37 on a uniform time grid $t_{D v}=v \Delta t, v=$ $0,1, \ldots$ assuming that within each interval $\left[t_{D v}, t_{D(v+1)}\right]$, the functions $c_{i j}\left(t_{D}\right)$ are constant and equal to some value $c_{i j}^{v}$ and $p_{D w u}\left(t_{D}\right)=p_{D w u}^{v}$. Finally, the system of integral equations given by Eqs. 37 and 37 will be reduced to the following system of linear algebraic equations:

$$
\begin{aligned}
& \sum_{i=0}^{K-1} \sum_{j=0}^{M-1} \sum_{v=0}^{V} c_{i j}^{v} F_{i j m k}^{V-v}=p_{D w u}^{V}, \\
& c_{00}^{V}=1,
\end{aligned}
$$

where

$$
F_{i j m k}^{V}=\int_{t_{D V}}^{t_{D(V+1)}} \Theta_{2 i}\left(1, \theta_{k}, \tau\right) \mathrm{Z}_{2 j}\left(z_{D m}, \tau\right) \mathrm{d} \tau .
$$

A technique for the fast and accurate evaluation of the matrix $F_{i j k m}^{V}$ is given in Appendix 1 . Due to the singular nature of $q_{D}$ expansion, to obtain high accuracy, only a small number of equations must be considered. $K$ and $M$, chosen from the interval 15 to 30 (depending on the value of $v$ ), provide at least 3-4 digits precision for the slot dimensionless pressure $p_{D w u}$. Also, Eq. 38 is valid only for a uniform time grid, which for some cases might be regarded as a limitation, that is why we propose to adapt it to a time grid with a variable step size. In particular, the following 4-point time scheme is found to be stable and computationally efficient. Consider a sequence of time "subgrids" in the form: $t_{D v_{d}}=v 2^{d}, v=0,1,2,3,4 ; d=d_{0}, d_{0}+1, d_{0}+2, \ldots, d_{\max }$, and let $v_{d}$ denote $v$-th point on $d$-th subgrid, then our time scheme can be formalized as follows:

\section{First iteration:}

$$
\sum_{i=0}^{K-1} \sum_{j=0}^{M-1} \sum_{v=0}^{V} c_{i j}^{v_{d_{0}}} F_{i j m k}^{(V-v)_{d_{0}}}=p_{D w u}^{V_{d_{0}}}, \quad c_{00}^{V_{d_{0}}}=1, V=0,1,2,3,
$$


d+1-th iteration $\left(d_{0} \ll d \ll d_{\max }-1\right)$ :

$$
\begin{aligned}
& c_{i j}^{0_{d+1}}=\frac{c_{i j}^{0_{d}}+c_{i j}^{1_{d}}}{2}, c_{i j}^{1_{d+1}}=\frac{c_{i j}^{2_{d}}+c_{i j}^{3_{d}}}{2}, \\
& F_{i j m k}^{0_{d+1}}=F_{i j m k}^{0_{d}}+F_{i j m k}^{1_{d}}, F_{i j m k}^{1_{d+1}}=F_{i j m k}^{2_{d}}+F_{i j m k}^{3_{d}}, \\
& \sum_{i=0}^{K-1} \sum_{j=0}^{M-1} \sum_{v=0}^{V} c_{i j}^{v_{d+1}} F_{i j m k}^{(V-v)_{d+1}}=p_{D w u}^{V_{d+1}}, \quad c_{00}^{V_{d+1}}=1, V=2,3 .
\end{aligned}
$$

Note that here we assume that $v_{d}+1 \equiv(v+1)_{d}$ in the definition of $F_{i j m k}^{V}$ (see Eq. 40). This procedure allows us to obtain the pressure change $p_{D w u}(t)$ and the coefficients $c_{i j}(t)$ on the gridpoints covering time range $2^{d_{0}}<t_{D}<2^{d_{\max }+2}$. Also in order to avoid missing near wellbore diffusion effects we need $t_{D \min }=2^{d_{0}}$ to be much less then $\max \left\{1, l_{D w}\right\}$. We suggest to chose $d_{0}=-10$ (and thus $t_{D \min } \approx 10^{-3}$ ) or less if earlier time data is required. The pressure and $c_{i j}$ values outside the time grid points can be obtained by simple logarthimic interpolation. We can also substitute obtained coefficients $c_{i j}(t)$ into Eq. 33 and easily obtain pressure (and its derivative) at any point of the reservoir.

\subsubsection{Pressure-Averaged Uniform-Flux Solution}

Next we present a simplified solution based on the assumption that the flux density is uniform on the slot. In this case, the mixed boundary value problem remains the same, except that Eq. 15 should be replaced with

$$
4 \lambda_{h} b l_{w} \frac{\partial \mathcal{P}}{\partial r}\left(r_{w}, \theta, z, t\right)=-q \text { for }|z|<l_{w} \text { and }\left|r_{w} \theta\right|<b,
$$

which can be rewritten in dimensionless form as

$$
\frac{\partial p_{D}}{\partial r_{D}}\left(1, \theta, z_{D}, t\right)=-\frac{\pi}{b_{D}} \text { for }\left|z_{D}\right|<1 \text { and }|\theta|<b_{D}
$$

Because Eq. 27 is valid for any flux density distribution, we can use it directly to obtain the pressure response

$$
p_{D}\left(r_{D}, \theta, z_{D}, t_{D}\right)=\frac{\pi}{b_{D}} \int_{0}^{t_{D}} \int_{0}^{1} \int_{0}^{b_{D}} \mathrm{G}\left(r_{D}, \theta, \psi, z_{D}, u, t_{D}-\tau\right) \mathrm{d} u \mathrm{~d} \psi \mathrm{d} \tau .
$$

Performing integration in the above equation yields

$$
p_{D}\left(r_{D}, \theta, z_{D}, t_{D}\right)=\frac{\pi}{b_{D}} \int_{0}^{t_{D}} \Theta_{q}\left(r_{D}, \theta, \tau\right) Z_{q}\left(z_{D}, \tau\right) \mathrm{d} \tau,
$$

where

$$
\Theta_{q}\left(r_{D}, \theta, t_{D}\right)=\mathcal{L}^{-1}\left\{\frac{1}{\sqrt{s}} \sum_{n=0}^{\infty}\left(1-\frac{\delta_{0 n}}{2}\right) \frac{2 \mathrm{~K}_{n}\left(r_{D} \sqrt{s}\right) \cos (n \theta)}{\mathrm{K}_{n+1}(\sqrt{s})+\mathrm{K}_{n-1}(\sqrt{s})} \frac{\sin \left(n b_{D}\right)}{n}\right\},
$$

and

$$
Z_{q}\left(z_{D}, \tau\right)=2\left[\operatorname{erf}\left(\frac{1+z_{D}}{2 v \sqrt{\tau}}\right)+\operatorname{erf}\left(\frac{1-z_{D}}{2 v \sqrt{\tau}}\right)\right]
$$


Now we can obtain the dimensionless wellbore pressure by taking the average value of Eq. 48 over the slot surface, which is equal to

$$
p_{D w a}\left(t_{D}\right)=\frac{1}{b_{D}^{2}} \int_{0}^{t_{D}} \hat{\Theta}_{q}(\tau) \hat{Z}_{q}(\tau) \mathrm{d} \tau
$$

with

$$
\hat{\Theta}_{q}\left(t_{D}\right)=\mathcal{L}^{-1}\left\{\frac{1}{\sqrt{s}} \sum_{n=0}^{\infty}\left(1-\frac{\delta_{0 n}}{2}\right) \frac{2 \mathrm{~K}_{n}(\sqrt{s})}{\mathrm{K}_{n+1}(\sqrt{s})+\mathrm{K}_{n-1}(\sqrt{s})} \frac{\sin ^{2}\left(n b_{D}\right)}{n^{2}}\right\},
$$

and

$$
\hat{Z}_{q}(\tau)=2 \operatorname{erf}\left(\frac{1}{v \sqrt{\tau}}\right)+2 \frac{v \sqrt{\tau}}{\sqrt{\pi}}\left(e^{-1 / v^{2} \tau}-1\right) .
$$

\subsection{Single-Slot Solution in a Vertically Bounded Medium}

The solution methodology presented above for the infinite $3 \mathrm{D}$ reservoir unbounded in the $z$ direction can be extended to the finite formation thickness case shown in Fig. 3. If we consider a reservoir with a thickness $h$ and a distance $z_{w}$ from the middle point of the slot to the bottom of the formation, then the dimensionless form of MBVP defined by Eqs. 13-18 will remain the same with an additional boundary condition (no-flow at the bottom and top boundaries), which can be written as

$$
\frac{\partial \mathcal{P}}{\partial z}\left(r_{w}, \theta,-z_{w}, t\right)=\frac{\partial \mathcal{P}}{\partial z}\left(r_{w}, \theta, h-z_{w}, t\right)=0,
$$

or in dimensionless form as

$$
\frac{\partial p_{D}}{\partial z_{D}}\left(1, \theta,-z_{D w}, t_{D}\right)=\frac{\partial p_{D}}{\partial z_{D}}\left(1, \theta, h_{D}-z_{D w}, t_{D}\right)=0,
$$

where

$$
h_{D}=\frac{h}{l_{w}} \text { and } \quad z_{D w}=\frac{z_{w}}{l_{w}} .
$$

Again we replace Eqs. 22 and 25 with the equivalent form

$$
\frac{\partial p_{D}}{\partial r_{D}}\left(1, \theta, z_{D}, t_{D}\right)=-q_{D}\left(\theta, z_{D}, t_{D}\right), \text { for }\left|z_{D}\right|<1 \text { and } 0 \leq \theta<b_{D},
$$

where the function $q_{D}\left(\theta, z_{D}, t_{D}\right)$ is unknown and will be specified later. Using the method of images, we can bring this problem back into the infinite domain by adding a set of slots with their middle points being positioned at $z_{D}= \pm 2 l h_{D}, l=1,2, \ldots, \infty$ with the same flux density distribution as on the original slot, and at $z_{D}= \pm 2 l h_{D}-2 z_{D w}, l=0,1,2, \ldots, \infty$ with the inverse flux density distribution $q_{D}\left(\theta,-z_{D}, t_{D}\right)$. Then the solution to the correponding boundary-value problem can be written as

$$
p_{D}\left(r_{D}, \theta, z_{D}, t_{D}\right)=\int_{0}^{t_{D}} \int_{-1}^{1} \int_{0}^{b_{D}} q_{D}(\psi, u, \tau) \mathrm{G}_{h}\left(r_{D}, \theta, \psi, z_{D}, u, t_{D}-\tau\right) \mathrm{d} u \mathrm{~d} \psi \mathrm{d} \tau,
$$

where $\mathrm{G}_{h}$ is a Green's function for the corresponding problem, which can be expressed as

$$
\mathrm{G}_{h}\left(r_{D}, \theta, \psi, z_{D}, u, t_{D}\right)=\Theta\left(r_{D}, \theta, \psi, t_{D}\right)\left[Z\left(z_{D}, u, t_{D}\right) / 2+Z_{h}\left(z_{D}, u, t_{D}\right)\right],
$$


with $\Theta\left(r_{D}, \theta, \psi, t_{D}\right)$ and $Z\left(z_{D}, u, t_{D}\right)$ being defined by Eqs. 29-30 and

$$
\begin{aligned}
\mathrm{Z}_{h}\left(z_{D}, u, t_{D}\right)= & \left.\frac{e^{-\frac{\left(z_{D}-u+2 z_{D w}\right)^{2}}{4 v^{2} t_{D}}}+\sum_{l=1}^{\infty}\left[\frac{e^{-\frac{\left(z_{D}-u-2 l h_{D}\right)^{2}}{4 v^{2} t_{D}}}}{\sqrt{\pi t_{D}} v}+\frac{e^{-\frac{\left(z_{D}-u+2 l h_{D}\right)^{2}}{4 v^{2} t_{D}}}}{\sqrt{\pi t_{D}} v}\right.}{\sqrt{\pi t_{D}} v}\right] . \\
& +\frac{(-1)^{l} e^{-\frac{\left(z_{D}-u+2 z_{D w}-2 l h_{D}\right)^{2}}{4 v^{2} t_{D}}}}{\sqrt{\pi t_{D}} v}+.
\end{aligned}
$$

As in the unbounded case, let us assume that the flux density $q_{D}\left(\theta, z_{D}, t_{D}\right)$ can be expanded in terms of Chebyshev polynomials as (note that since $q_{D}$ is no longer an even function of $z_{D}$ we must include both even and odd Chebyshev Polynomials)

$$
q_{D}(\theta, u, \tau)=\frac{1}{\pi} \sum_{i=0}^{\infty} \sum_{j=0}^{\infty} c_{i j}(t) \frac{(-1)^{i} T_{2 i}\left(\theta / b_{D}\right)}{\sqrt{b_{D}^{2}-\theta^{2}}} \frac{T_{j}\left(z_{D}\right)}{\sqrt{1-z_{D}^{2}}},
$$

then we obtain the following expression for the dimensionless pressure distribution in the reservoir:

$$
\begin{aligned}
p_{D}\left(r_{D}, \theta, z_{D}, t_{D}\right)= & \sum_{i=0}^{\infty} \sum_{j=0}^{\infty} \int_{0}^{t_{D}} c_{i j}(\tau) \Theta_{2 i}\left(r_{D}, \theta, t_{D}-\tau\right) \\
& \times\left[\mathrm{Z}_{j}\left(z_{D}, t_{D}-\tau\right)+\mathrm{Z}_{h j}\left(z_{D}, t_{D}-\tau\right)\right] \mathrm{d} \tau,
\end{aligned}
$$

with $\Theta_{2 i}$ and $Z_{j}$ being defined by Eqs. 34-35 and

$$
\mathrm{Z}_{h j}\left(z_{D}, t_{D}\right)=\int_{0}^{\pi} \mathrm{Z}_{h}\left(z_{D}, \cos (\eta), t_{D}\right) \cos (j \eta) \mathrm{d} \eta .
$$

As in the previous case, a method is given in Appendix 1 for the efficient computation of $\mathrm{Z}_{h j}$. Finally, to determine unknown functions $c_{i j}\left(t_{D}\right)$ and $p_{D w}\left(t_{D}\right)$, we need to solve the following system of integral equations:

$$
\begin{gathered}
\sum_{i=0}^{\infty} \sum_{j=0}^{\infty} \int_{0}^{t_{D}} c_{i j}(\tau) \Theta_{2 i}\left(1, \theta, t_{D}-\tau\right) \mathrm{Z}_{h j}\left(z_{D}, t_{D}-\tau\right) \mathrm{d} \tau \\
=p_{D w u}\left(t_{D}\right) \text { for }\left|z_{D}\right|<1 \text { and } 0 \leq \theta<b_{D}, \\
c_{00}\left(t_{D}\right)=1,
\end{gathered}
$$

which can be treated in the same way as we proposed in the vertically unbounded case.

\subsubsection{Pressure-Averaged Uniform-Flux Solution}

Let us also present a simplified solution based on the assumption that the flux density is uniform on the slot (see Eqs. 45 and 46). The pressure response can be easily obtained from Eq. 58 as

$$
p_{D}\left(r_{D}, \theta, z_{D}, t_{D}\right)=\frac{\pi}{b_{D}} \int_{0}^{t_{D}} \int_{0}^{1} \int_{0}^{b_{D}} \mathrm{G}_{\mathrm{h}}\left(r_{D}, \theta, \psi, z_{D}, u, t_{D}-\tau\right) \mathrm{d} u \mathrm{~d} \psi \mathrm{d} \tau .
$$


Performing integration in the above equation yields

$$
p_{D}\left(r_{D}, \theta, z_{D}, t_{D}\right)=\frac{\pi}{b_{D}} \int_{0}^{t_{D}} \Theta_{q}\left(r_{D}, \theta, \tau\right) Z_{q h}\left(z_{D}, \tau\right) \mathrm{d} \tau,
$$

where $\Theta_{q}$ is defined by Eq. 49 and

$$
\begin{aligned}
Z_{q h}\left(z_{D}, \tau\right)= & \sum_{i=-\infty}^{\infty}\left[\operatorname{erf}\left(\frac{1+z_{D}+2 i h_{D}}{2 v \sqrt{\tau}}\right)+\operatorname{erf}\left(\frac{1-\left(z_{D}+2 i h_{D}\right)}{2 v \sqrt{\tau}}\right)\right. \\
& \left.+\operatorname{erf}\left(\frac{1+z_{D}+2 z_{D w}+2 i h_{D}}{2 v \sqrt{\tau}}\right)+\operatorname{erf}\left(\frac{1-\left(z_{D}+2 z_{D w}+2 i h_{D}\right)}{2 v \sqrt{\tau}}\right)\right] .
\end{aligned}
$$

Now we can estimate the dimensionless wellbore pressure by taking the average value of Eq. 67 over the slot surface, which is equal to

$$
p_{D w a}\left(t_{D}\right)=\frac{1}{b_{D}^{2}} \int_{0}^{t_{D}} \hat{\Theta}_{q}(\tau) \hat{Z}_{q h}(\tau) \mathrm{d} \tau
$$

with $\hat{\Theta}_{q}$ defined by Eq. 52 and

$$
\hat{Z}_{q h}(\tau)=W\left(\tau, 0, h_{D}\right)+W\left(\tau, z_{D w}, h_{D}\right)
$$

where

$$
\begin{aligned}
W\left(\tau, x, h_{D}\right)= & \sum_{i=-\infty}^{\infty}\left(v \sqrt{\frac{\tau}{\pi}}\left[e^{-\frac{\left(x+i h_{D}+1\right)^{2}}{v^{2} \tau}}+e^{-\frac{\left(x+i h_{D}-1\right)^{2}}{v^{2} \tau}}-2 e^{-\frac{\left(x+i h_{D}\right)^{2}}{v^{2} \tau}}\right]+\left(x+i h_{D}+1\right)\right. \\
& \times \operatorname{erf}\left(\frac{x+i h_{D}+1}{v \sqrt{\tau}}\right)+\left(x+i h_{D}-1\right) \operatorname{erf}\left(\frac{x+i h_{D}-1}{v \sqrt{\tau}}\right) \\
& \left.-2\left(x+i h_{D}\right) \operatorname{erf}\left(\frac{x+i h_{D}}{v \sqrt{\tau}}\right)\right) .
\end{aligned}
$$

\subsection{Solutions for Vertical Multiple Slots}

Another generalization is to consider a number of same slots symmetrically placed on the wellbore, as shown in Fig. 3. In this case the mixed boundary value problem will remain the same, as given by Eqs. 13-17, and 54 with an additional inner boundary condition

$$
\frac{\partial \mathcal{P}}{\partial \theta}\left(r_{w},-\frac{\pi}{N_{o}}, z, t\right)=\frac{\partial \mathcal{P}}{\partial \theta}\left(r_{w}, \frac{\pi}{N_{o}}, z, t\right)=0 .
$$

which defines the symmetry with respect to variable $\theta$ due to $N_{o}$ symmetrically placed slots. The boundary condition on the total flowrate Eq. 18 should be also replaced with

$$
r_{w} \lambda_{h} \int_{-b / r_{w}}^{b / r_{w}} \int_{-l_{w}}^{l_{w}} \frac{\partial \mathcal{P}}{\partial r}\left(r_{w}, \theta, z, t\right) \mathrm{d} z \mathrm{~d} \theta=-q / N_{o} .
$$

Using the same dimensionless variables as before (Eqs. 19 and 56), these equations can be written in dimensionless form as

$$
\begin{aligned}
& \frac{\partial p_{D}}{\partial \theta}\left(1,-\frac{\pi}{N_{o}}, z_{D}, t_{D}\right)=\frac{\partial p_{D}}{\partial \theta}\left(1, \frac{\pi}{N_{o}}, z_{D}, t_{D}\right)=0, \\
& \frac{1}{2 \pi} \int_{0}^{b_{D}} \int_{-1}^{1} \frac{\partial p_{D}}{\partial r_{D}}\left(1, \theta, z_{D}, t_{D}\right) \mathrm{d} z_{D}=-1 / N_{o} .
\end{aligned}
$$


As in the previous cases,we assume that the flux density on each slot is expressed in terms of a series of Chebyshev polynomials as

$$
q_{D}(\theta, u, \tau)=\frac{1}{\pi} \sum_{i=0}^{\infty} \sum_{j=0}^{\infty} c_{i j}(t) \frac{(-1)^{i} T_{2 i}\left(\theta / b_{D}\right)}{\sqrt{b_{D}^{2}-\theta^{2}}} \frac{T_{j}\left(z_{D}\right)}{\sqrt{1-z_{D}^{2}}}
$$

then the solution for the dimensionless pressure $p_{D}$ can be obtained in the following form

$$
p_{D}\left(r_{D}, \theta, z_{D}, t_{D}\right)=\int_{0}^{t_{D}} \int_{-1}^{1} \int_{0}^{b_{D}} q_{D}(\psi, u, \tau) \mathrm{G}_{h N_{o}}\left(r_{D}, \theta, \psi, z_{D}, u, t_{D}-\tau\right) \mathrm{d} u \mathrm{~d} \psi \mathrm{d} \tau,
$$

where $\mathrm{G}_{h N_{o}}$ is a Green's function for the corresponding problem, which can be expressed as

$$
\mathrm{G}_{h N_{o}}\left(r_{D}, \theta, \psi, z_{D}, u, t_{D}\right)=\Theta_{N_{o}}\left(r_{D}, \theta, \psi, t_{D}\right)\left[Z\left(z_{D}, u, t_{D}\right) / 2+Z_{h}\left(z_{D}, u, t_{D}\right)\right],
$$

with

$\Theta_{N_{o}}\left(r_{D}, \theta, \psi, t_{D}\right)=\mathcal{L}^{-1}\left\{\frac{1}{\sqrt{s}} \sum_{n=0}^{\infty}\left(1-\frac{\delta_{0 n}}{2}\right) \frac{2 \mathrm{~K}_{n N_{o}}\left(r_{D} \sqrt{s}\right) \cos \left(n N_{o} \theta\right) \cos \left(n N_{o} \psi\right)}{\mathrm{K}_{n N_{o}+1}(\sqrt{s})+\mathrm{K}_{n N_{o}-1}(\sqrt{s})}\right\}$,

and $Z\left(z_{D}, u, t_{D}\right)$ and $Z_{h}\left(z_{D}, u, t_{D}\right)$ being defined by Eqs. 30 and 60, respectively. The substitution of $q_{D}$ given by Eq. 76 in Eq. 77 yields

$$
\begin{aligned}
p_{D}\left(r_{D}, \theta, z_{D}, t_{D}\right)= & \sum_{i=0}^{\infty} \sum_{j=0}^{\infty} \int_{0}^{t_{D}} c_{i j}(\tau) \Theta_{N_{o} 2 i}\left(r_{D}, \theta, t_{D}-\tau\right)\left[\mathrm{Z}_{j}\left(z_{D}, t_{D}-\tau\right)\right. \\
& \left.+\mathrm{Z}_{h j}\left(z_{D}, t_{D}-\tau\right)\right] \mathrm{d} \tau
\end{aligned}
$$

where

$$
\Theta_{N_{o} 2 i}\left(r_{D}, \theta, t_{D}\right)=\mathcal{L}^{-1}\left\{\frac{1}{\sqrt{s}} \sum_{n=0}^{\infty}\left(1-\frac{\delta_{0 n}}{2}\right) \frac{2 \mathrm{~K}_{n N_{o}}\left(r_{D} \sqrt{s}\right) \mathrm{J}_{2 i}\left(n N_{o} b_{D}\right) \cos \left(n N_{o} \psi\right)}{\mathrm{K}_{n N_{o}+1}(\sqrt{s})+\mathrm{K}_{n N_{o}-1}(\sqrt{s})}\right\} .
$$

Finally, the unknown functions $c_{i j}\left(t_{D}\right)$ and $p_{D w}\left(t_{D}\right)$ can be determined by solving the following system of integral equations:

$$
\begin{gathered}
\sum_{i=0}^{\infty} \sum_{j=0}^{\infty} \int_{0}^{t_{D}} c_{i j}(\tau) \Theta_{N_{o} 2 i}\left(1, \theta, t_{D}-\tau\right) Z_{h j}\left(z_{D}, t_{D}-\tau\right) \mathrm{d} \tau \\
=p_{D w u}\left(t_{D}\right) \text { for }\left|z_{D}\right|<1 \text { and } 0 \leq \theta<b_{D} \\
c_{00}\left(t_{D}\right)=1 .
\end{gathered}
$$

The solution of this system can be obtained by using the same technique as in previous cases. 


\subsubsection{Pressure-Averaged Uniform-Flux Solution}

Next we present an approximate solution based on the assumption that the flux density is uniform on the slots. In this case, the mixed boundary value problem remains the same, except that Eqs. 15 and 73 should be replaced with

$$
4 \lambda_{h} b l_{w} \frac{\partial \mathcal{P}}{\partial r}\left(r_{w}, \theta, z, t\right)=-q / N_{0} \text { for }|z|<l_{w} \text { and }\left|r_{w} \theta\right|<b,
$$

which can be rewritten in dimensionless form as

$$
\frac{\partial p_{D}}{\partial r_{D}}\left(1, \theta, z_{D}, t\right)=-\frac{\pi}{b_{D} N_{0}} \text { for }\left|z_{D}\right|<1 \text { and }|\theta|<b_{D} .
$$

Again, since Eq. 77 is valid for any flux density distribution, we can use it to directly obtain the pressure response as

$$
p_{D}\left(r_{D}, \theta, z_{D}, t_{D}\right)=\frac{\pi}{b_{D} N_{0}} \int_{0}^{t_{D}} \int_{-1}^{1} \int_{0}^{b_{D}} \mathrm{G}_{h N_{o}}\left(r_{D}, \theta, \psi, z_{D}, u, t_{D}-\tau\right) \mathrm{d} u \mathrm{~d} \psi \mathrm{d} \tau .
$$

Performing integration in the above equation yields

$$
p_{D}\left(r_{D}, \theta, z_{D}, t_{D}\right)=\frac{\pi}{b_{D} N_{0}} \int_{0}^{t_{D}} \Theta_{q N_{0}}\left(r_{D}, \theta, \tau\right) Z_{q h}\left(z_{D}, \tau\right) \mathrm{d} \tau,
$$

where

$\Theta_{q N_{o}}\left(r_{D}, \theta, t_{D}\right)=\mathcal{L}^{-1}\left\{\frac{1}{\sqrt{s}} \sum_{n=0}^{\infty}\left(1-\frac{\delta_{0 n}}{2}\right) \frac{2 \mathrm{~K}_{n N_{o}}\left(r_{D} \sqrt{s}\right) \cos \left(n N_{o} \theta\right)}{\mathrm{K}_{n N_{o}+1}(\sqrt{s})+\mathrm{K}_{n N_{o}-1}(\sqrt{s})} \frac{\sin \left(n N_{o} b_{D}\right)}{n N_{0}}\right\}$,

and $Z_{q h}$ is defined by Eq. 68 .

Now we can obtain the dimensionless wellbore pressure by taking the average value of Eq. 87 over the slot surface, which is equal to

$$
p_{D w a}\left(t_{D}\right)=\frac{1}{b_{D}^{2}} \int_{0}^{t_{D}} \hat{\Theta}_{q N_{0}}(\tau) \hat{Z}_{q h}(\tau) \mathrm{d} \tau
$$

with

$$
\hat{\Theta}_{q N_{o}}\left(t_{D}\right)=\mathcal{L}^{-1}\left\{\frac{1}{\sqrt{s}} \sum_{n=0}^{\infty}\left(1-\frac{\delta_{0 n}}{2}\right) \frac{2 \mathrm{~K}_{n N_{o}}(\sqrt{s})}{\mathrm{K}_{n N_{o}+1}(\sqrt{s})+\mathrm{K}_{n N_{o}-1}(\sqrt{s})} \frac{\sin ^{2}\left(n N_{o} b_{D}\right)}{n^{2} N_{0}^{2}}\right\},
$$

and $\hat{Z}_{q h}$ defined by Eq. 70 .

\subsection{Vertical Slot Solutions in 3D Anisotropic Porous Media}

In this section, we generalize the solution proposed above to the case of $k_{x} \neq k_{y}\left(\lambda_{y} \neq \lambda_{x}\right)$, i.e., the permeabilities in the $x$ and $y$ directions are different. Here we assume that $\lambda_{y}>\lambda_{x}$. If it is not the case, we can always rotate the coordinate system in the horizontal plane and interchange the $x$ and $y$ axis. Let us denote $\chi=\sqrt{\lambda_{x} / \lambda_{y}}$, make a coordinate transformation $y \rightarrow y^{\prime}=y / \chi$, and introduce the elliptical coordinates in the horizontal plane

$$
\begin{aligned}
& x / r_{w}=a \cosh \xi \cos \theta, \\
& y^{\prime} / r_{w}=a \sinh \xi \sin \theta,
\end{aligned}
$$


with $a^{2}=1-\chi^{2}$, then we can write the corresponding boundary-value problem as

$$
\begin{aligned}
& \frac{2 \lambda_{x}}{r_{w}^{2} a^{2}(\cosh (2 \xi)-\cos (2 \theta))}\left(\frac{\partial^{2} \mathcal{P}}{\partial \xi^{2}}+\frac{\partial^{2} \mathcal{P}}{\partial \theta^{2}}\right)+\lambda_{v} \frac{\partial^{2} \mathcal{P}}{\partial z^{2}}=\varphi \frac{\partial \mathcal{P}}{\partial t}, \\
& \mathcal{P}(\xi, \theta, z, 0)=0, \\
& \mathcal{P}\left(\xi_{w}, z, \theta, t\right)=\mathcal{P}_{w u}(t), \text { for }|z|<l_{w} \text { and } \theta \in \bigcup_{p} \Lambda_{p}, \\
& \frac{\partial \mathcal{P}}{\partial \xi}\left(\xi_{w}, \theta, z, t\right)=0, \text { for }|z| \geq l_{w} \text { or } \theta \notin \bigcup_{p} \Lambda_{p}, \\
& \mathcal{P}(\xi, z, \theta, t) \rightarrow 0 \text { as } \xi \rightarrow \infty, \\
& \frac{\partial \mathcal{P}}{\partial z}\left(\xi_{w}, \theta,-z_{w}, t\right)=\frac{\partial \mathcal{P}}{\partial z}\left(\xi_{w}, \theta, h-z_{w}, t\right)=0, \\
& r_{w} \sqrt{\lambda_{x} \lambda_{y}} \sum_{p} \int_{\Lambda_{p}} \int_{-l_{w}}^{l_{w}} \frac{\partial \mathcal{P}}{\partial \xi}\left(\xi_{w}, \theta, z, t\right) \mathrm{d} z \mathrm{~d} \theta=-q,
\end{aligned}
$$

where $\xi_{w}=\operatorname{arctanh}(\chi)$ and $\Lambda_{p}=\left\{\theta:\left|\theta-\theta_{p}\right| \leq b / r_{w}=b_{D}\right\}$ with $\theta_{p}$ being the angular coordinate of the center of the $p$-th slot, as shown in Fig. 3. Note that in this case we do not have symmetry any more with respect to $\theta$ due to anisotropy in the $x-y$ (horizontal) plane. Using the dimensionless variables introduced earlier in Eq. 19 and 56 (except that due to horizontal anisotropy $k_{h}$ should be replaced with $k_{x}$ ), the mixed boundary value problem in a $3 \mathrm{D}$ anisotropic porous medium can be written as

$$
\begin{aligned}
& \frac{2}{a^{2}(\cosh (2 \xi)-\cos (2 \theta))}\left(\frac{\partial^{2} p_{D}}{\partial \xi^{2}}+\frac{\partial^{2} p_{D}}{\partial \theta^{2}}\right)+v^{2} \frac{\partial^{2} p_{D}}{\partial z_{D}^{2}}=\frac{\partial p_{D}}{\partial t_{D}}, \\
& p_{D}\left(\xi, \theta, z_{D}, 0\right)=0, \\
& p_{D}\left(\xi_{w}, z_{D}, \theta, t_{D}\right)=p_{D w u}(t), \text { for }\left|z_{D}\right|<1 \text { and } \theta \in \bigcup_{p} \Lambda_{p}, \\
& \frac{\partial p_{D}}{\partial \xi}\left(\xi_{w}, \theta, z_{D}, t_{D}\right)=0, \text { for }\left|z_{D}\right| \geq 1 \text { or } \theta \notin \bigcup_{p} \Lambda_{p}, \\
& p_{D}\left(\xi, z_{D}, \theta, t_{D}\right) \rightarrow 0 \text { as } \xi \rightarrow \infty, \\
& \frac{\partial p_{D}}{\partial z_{D}}\left(\xi_{w}, \theta,-z_{D w}, t_{D}\right)=\frac{\partial p_{D}}{\partial z_{D}}\left(\xi_{w}, \theta, h_{D}-z_{D w}, t_{D}\right)=0, \\
& \frac{1}{4 \pi \chi} \sum_{p} \int_{\Lambda_{p}} \int_{-1}^{1} \frac{\partial p_{D}}{\partial \xi}\left(\xi_{w}, \theta, z_{D}, t_{D}\right) \mathrm{d} z_{D} \mathrm{~d} \theta=-1 .
\end{aligned}
$$

As in previous cases, we assume that on the $p$-th slot the flux density $q_{D}\left(\theta, z_{D}, t_{D}\right)$ can be expanded in terms of the Chebyshev polynomials as

$$
q_{D}^{p}\left(\theta, z_{D}, t_{D}\right)=\frac{\chi}{\pi} \sum_{i=0}^{\infty} \sum_{j=0}^{\infty} c_{i j}^{p}\left(t_{D}\right) \frac{(-1)^{[i / 2]} T_{i}\left(\left[\theta-\theta_{p}\right] / b_{D}\right)}{\sqrt{b_{D}^{2}-\left(\theta-\theta_{p}\right)^{2}}} \frac{T_{j}\left(z_{D}\right)}{\sqrt{1-z_{D}^{2}}},
$$

then the corresponding pressure drop induced by such a flux density distribution can be written as

$$
p_{D}^{p}\left(\xi, \theta, z_{D}, t_{D}\right)=\int_{0}^{t_{D}} \int_{-1}^{1} \int_{-b_{D}}^{b_{D}} q_{D}^{p}(\psi, u, \tau) \mathrm{G}_{h}^{p}\left(\xi, \theta, \psi, z_{D}, u, t_{D}-\tau\right) \mathrm{d} u \mathrm{~d} \psi \mathrm{d} \tau,
$$


where

$$
\mathrm{G}_{h}^{p}\left(\xi, \theta, \psi, z_{D}, u, t_{D}\right)=\Theta^{p}\left(\xi, \theta, \psi, t_{D}\right)\left[Z\left(z_{D}, u, t_{D}\right) / 2+Z_{h}\left(z_{D}, u, t_{D}\right)\right] .
$$

and

$$
\begin{aligned}
\Theta^{p}\left(\xi, \theta, \psi, t_{D}\right)= & \frac{1}{\chi} \mathcal{L}^{-1}\left\{\sum_{r=0}^{\infty} \frac{\operatorname{Mc}_{r}\left(\xi,-a^{2} s / 4\right)}{\operatorname{Mc}_{r}^{\prime}\left(\xi_{w},-a^{2} s / 4\right)} \operatorname{ce}_{r}\left(\theta,-a^{2} s / 4\right) \mathrm{ce}_{r}\left(\psi,-a^{2} s / 4\right)\right. \\
& \left.+\sum_{r=1}^{\infty} \frac{\operatorname{Ms}_{r}\left(\xi,-a^{2} s / 4\right)}{\operatorname{Ms}_{r}^{\prime}\left(\xi_{w},-a^{2} s / 4\right)} \operatorname{se}_{r}\left(\theta,-a^{2} s / 4\right) \mathrm{se}_{r}\left(\psi,-a^{2} s / 4\right)\right\}
\end{aligned}
$$

$\mathrm{ce}_{n}$ and $\mathrm{se}_{n}$ are being normalized angular even and odd Mathieu functions, and $\mathrm{Mc}_{n}$ and $\mathrm{Ms}_{n}$ are radial even and odd Mathieu functions of the third kind. Expanding Mathieu functions over sines and cosines we can rewrite the last expression as

$\Theta^{p}\left(\xi, \theta, \psi, t_{D}\right)=\mathcal{L}^{-1}\left\{\sum_{n=0}^{\infty} F c_{n}\left(\xi, \theta, a^{2} s / 4\right) \cos (n \psi)+\sum_{n=1}^{\infty} F s_{n}\left(\xi, \theta, a^{2} s / 4\right) \sin (n \psi)\right\}$,

where

$$
\begin{aligned}
& F c_{2 n+\gamma}\left(\xi, \theta, a^{2} s / 4\right)=\sum_{r=0}^{\infty} \frac{\operatorname{Mc}_{2 r+\gamma}\left(\xi,-a^{2} s / 4\right)}{\operatorname{Mc}_{2 r+\gamma}^{\prime}\left(\xi_{w},-a^{2} s / 4\right)} A_{2 n+\gamma}^{2 r+\gamma} \operatorname{ce}_{2 r+\gamma}\left(\theta,-a^{2} s / 4\right), \gamma=0,1, \\
& F s_{2 n+\gamma}\left(\xi, \theta, a^{2} s / 4\right)=\sum_{r=0}^{\infty} \frac{\mathrm{Ms}_{2 r+\gamma}\left(\xi,-a^{2} s / 4\right)}{\operatorname{Ms}_{2 r+\gamma}^{\prime}\left(\xi_{w},-a^{2} s / 4\right)} B_{2 n+\gamma}^{2 r+\gamma} \operatorname{se}_{2 r+\gamma}\left(\theta,-a^{2} s / 4\right), \gamma=0,1
\end{aligned}
$$

and $A_{2 n+\gamma}^{2 r+\gamma}$ and $B_{2 n+\gamma}^{2 r+\gamma}$ are corresponding expansion coefficients. The substitution of $q_{D}^{p}$ given by Eq. 107 in Eq. 108 yields

$$
\begin{aligned}
p_{D}^{p}\left(r_{D}, \theta, z_{D}, t_{D}\right)= & \sum_{i=0}^{\infty} \sum_{j=0}^{\infty} \int_{0}^{t_{D}} c_{i j}^{p}\left(t_{D}\right) \Theta_{i}^{p}\left(\xi, \theta, t_{D}-\tau\right)\left[\mathrm{Z}_{j}\left(z_{D}, t_{D}-\tau\right)\right. \\
& \left.+\mathrm{Z}_{h j}\left(z_{D}, t_{D}-\tau\right)\right] \mathrm{d} \tau
\end{aligned}
$$

where

$$
\begin{aligned}
& \Theta_{2 i}^{p}\left(\xi, \theta, t_{D}\right) \\
& =\mathcal{L}^{-1}\left\{\sum_{n=0}^{\infty}\left[F c_{n}\left(\xi, \theta, a^{2} s / 4\right) \cos \left(n \theta_{p}\right)+F s_{n}\left(\xi, \theta, a^{2} s / 4\right) \sin \left(n \theta_{p}\right)\right] \mathrm{J}_{2 i}\left(n b_{D}\right)\right\}, \\
& \Theta_{2 i+1}^{p}\left(\xi, \theta, t_{D}\right) \\
& \quad=\mathcal{L}^{-1}\left\{\sum_{n=1}^{\infty}\left[F s_{n}\left(\xi, \theta, a^{2} s / 4\right) \cos \left(n \theta_{p}\right)-F c_{n}\left(\xi, \theta, a^{2} s / 4\right) \sin \left(n \theta_{p}\right)\right] \mathrm{J}_{2 i+1}\left(n b_{D}\right)\right\} .
\end{aligned}
$$


Finally the unknown coefficients $c_{i j}\left(t_{D}\right)$ and $p_{D w u}$ can be obtained by solving the following system of integral equations:

$$
\begin{gathered}
\sum_{p} \sum_{i=0}^{\infty} \sum_{j=0}^{\infty} \int_{0}^{t_{D}} c_{i j}^{p}(\tau) \Theta_{i}^{p}\left(\xi_{w}, \theta, t_{D}-\tau\right) \mathrm{Z}_{h j}\left(z_{D}, t_{D}-\tau\right) \mathrm{d} \tau=p_{D w u}\left(t_{D}\right) \\
\text { for }\left|z_{D}\right|<1 \text { and } \theta \in \bigcup_{p} \Lambda_{p} \\
\qquad \sum_{p} c_{00}^{p}\left(t_{D}\right)=1
\end{gathered}
$$

which can be treated in the same way as we suggested previously. The only difficulty that might arise is the summation of the series in $\Theta_{i}^{p}$; we proposed one possible approach to solving this problem in Appendix 1. Now let us simplify the function $\Theta_{i}^{p}$ and the system of equations given by Eqs. 117 and 118 for a few particular cases.

\subsection{One Slot with its Center Positioned at $\theta=0, \pi$}

In this case, due to the obvious symmetry with respect to $\pm \theta$, we should consider only even Chebyshev polynomials in flux density expansion given by Eq. 107. Thus, the only non-zero coefficients are $c_{2 i j}^{1}$, and the function $\Theta_{2 i}^{1}$ smplifies to

$$
\Theta_{2 i}^{1}\left(\xi, \theta, t_{D}\right)=\mathcal{L}^{-1}\left\{\sum_{n=0}^{\infty} F c_{n}\left(\xi, \theta, a^{2} s / 4\right) \mathbf{J}_{2 i}\left(n b_{D}\right)\right\},
$$

and the system of integral equations to be solved becomes

$$
\begin{gathered}
\sum_{i=0}^{\infty} \sum_{j=0}^{\infty} \int_{0}^{t_{D}} c_{2 i j}^{1}(\tau) \Theta_{2 i}^{1}\left(\xi_{w}, \theta, t_{D}-\tau\right) \mathrm{Z}_{h j}\left(z_{D}, t_{D}-\tau\right) \mathrm{d} \tau=p_{D w u}\left(t_{D}\right) \\
\text { for }\left|z_{D}\right|<1 \text { and } \theta \in\left[0, b_{D}\right] \\
\qquad c_{00}^{1}\left(t_{D}\right)=1 .
\end{gathered}
$$

\subsubsection{One Slot with its Center Positioned at $\theta= \pm \frac{\pi}{2}$}

Here, due to the obvious symmetry around $\theta= \pm \pi / 2$, we should consider only the even Chebyshev polynomials in flux density expansion Eq. 107, thus the only non-zero coefficients are $c_{2 i j}^{1}$ and the function $\Theta_{2 i}^{1}$ simplifies to

$$
\begin{aligned}
& \Theta_{2 i}^{1}\left(\xi, \theta, t_{D}\right) \\
& =\mathcal{L}^{-1}\left\{\sum_{n=0}^{\infty}(-1)^{n}\left[F c_{2 n}\left(\xi, \theta, \frac{a^{2} s}{4}\right) \mathrm{J}_{2 i}\left(2 n b_{D}\right) \pm F s_{2 n+1}\left(\xi, \theta, \frac{a^{2} s}{4}\right) \mathrm{J}_{2 i}\left([2 n+1] b_{D}\right)\right]\right\}
\end{aligned}
$$


and the system of integral equations to be solved becomes

$$
\begin{gathered}
\sum_{i=0}^{\infty} \sum_{j=0}^{\infty} \int_{0}^{t_{D}} c_{2 i j}^{1}(\tau) \Theta_{2 i}^{1}\left(\xi_{w}, \theta, t_{D}-\tau\right) \mathrm{Z}_{h j}\left(z_{D}, t_{D}-\tau\right) \mathrm{d} \tau=p_{D w u}\left(t_{D}\right) \\
\text { for }\left|z_{D}\right|<1 \text { and } \theta \in\left[ \pm \frac{\pi}{2}, \pm \frac{\pi}{2}+b_{D}\right] \\
\qquad c_{00}^{1}\left(t_{D}\right)=1 .
\end{gathered}
$$

\subsubsection{Two Slots with Centers Positioned at $\theta=0$ and $\theta=\pi$}

Here, we can consider only one half of the problem in the domain $-\pi / 2 \leq \theta \leq \pi / 2$, and take only the symmetric part of $\Theta_{2 i}^{1}$ :

$$
\Theta_{s 2 i}^{1}\left(\xi, \theta, t_{D}\right)=\mathcal{L}^{-1}\left\{\sum_{n=0}^{\infty} F c_{2 n}\left(\xi, \theta, a^{2} s / 4\right) \mathrm{J}_{2 i}\left(2 n b_{D}\right)\right\},
$$

thus the system of integral equations to be solved becomes

$$
\begin{gathered}
\sum_{i=0}^{\infty} \sum_{j=0}^{\infty} \int_{0}^{t_{D}} c_{2 i j}^{1}(\tau) \Theta_{s 2 i}^{1}\left(\xi_{w}, \theta, t_{D}-\tau\right) \mathrm{Z}_{h j}\left(z_{D}, t_{D}-\tau\right) \mathrm{d} \tau=p_{D w u}\left(t_{D}\right) \\
\text { for }\left|z_{D}\right|<1 \text { and } \theta \in\left[0, b_{D}\right], \\
\qquad c_{00}^{1}\left(t_{D}\right)=\frac{1}{2} .
\end{gathered}
$$

\subsubsection{Two Slots with Centers Positioned at $\theta= \pm \frac{\pi}{2}$}

As in the previous case, we can consider only one half of the problem in the domain $0 \leq \theta \leq$ $\pi$, and thus take only the symmetric part of $\Theta_{2 i}^{1}$ :

$$
\Theta_{s 2 i}^{1}\left(\xi, \theta, t_{D}\right)=\mathcal{L}^{-1}\left\{\sum_{n=0}^{\infty}(-1)^{n} F c_{2 n}\left(\xi, \theta, a^{2} s / 4\right) \mathbf{J}_{2 i}\left(2 n b_{D}\right)\right\},
$$

and the system of integral equations to be solved becomes

$$
\begin{aligned}
& \sum_{i=0}^{\infty} \sum_{j=0}^{\infty} \int_{0}^{t_{D}} c_{2 i j}^{1}(\tau) \Theta_{s 2 i}^{1}\left(\xi_{w}, \theta, t_{D}-\tau\right) \mathrm{Z}_{h j}\left(z_{D}, t_{D}-\tau\right) \mathrm{d} \tau=p_{D w u}\left(t_{D}\right) \\
& \quad \text { for }\left|z_{D}\right|<1 \text { and } \theta \in\left[\frac{\pi}{2}, \frac{\pi}{2}+b_{D}\right] \\
& c_{00}^{1}\left(t_{D}\right)=\frac{1}{2}
\end{aligned}
$$


Table 1 Formation and fluid properties for the 4-slot packer module example

\begin{tabular}{|c|c|c|c|c|c|c|c|c|c|c|c|}
\hline$\phi_{m}$ & fraction & 0.2 & $\mu$ & $\mathrm{cp}$ & 2.5 & $c_{t}$ & $\mathrm{psi}^{-1}$ & $7 \times 10^{-6}$ & $\mathrm{q}$ & $\mathrm{RB} / \mathrm{D}$ & 5.43 \\
\hline$k_{h}$ & $\mathrm{mD}$ & 100 & $k_{v}$ & $\mathrm{mD}$ & 100 & $h$ & $\mathrm{ft}$ & 164.0 & $z_{w}$ & $\mathrm{ft}$ & 82.0 \\
\hline$l_{w}^{\S}$ & $\mathrm{ft}$ & $0.31(9.4 \mathrm{~cm})$ & $r_{w}$ & $\mathrm{ft}$ & 0.354 & $b^{\dagger}$ & $\mathrm{ft}$ & $0.12(3.725 \mathrm{~cm})$ & $S$ & & 5 \\
\hline$C_{D}$ & & 0 & & & & & & & & & \\
\hline
\end{tabular}

$b^{\dagger}$ is the half slot aperture and $l_{w}^{\S}$ is the half slot height (see Fig. 3)

\subsubsection{Four Slots with Centers Placed at $\theta=0, \theta=\pi$, and $\theta= \pm \frac{\pi}{2}$}

This is a combination of two previous cases. Again, it is enough to consider only two slots with $\theta_{1}=0$ and $\theta_{2}=\pi / 2$ and to take the symmetrical parts of the corresponding functions:

$$
\begin{aligned}
& \Theta_{s 2 i}^{1}\left(\xi, \theta, t_{D}\right)=\mathcal{L}^{-1}\left\{\sum_{n=0}^{\infty} F c_{2 n}\left(\xi, \theta, a^{2} s / 4\right) \mathrm{J}_{2 i}\left(2 n b_{D}\right)\right\}, \\
& \Theta_{s 2 i}^{2}\left(\xi, \theta, t_{D}\right)=\mathcal{L}^{-1}\left\{\sum_{n=0}^{\infty}(-1)^{n} F c_{2 n}\left(\xi, \theta, a^{2} s / 4\right) \mathrm{J}_{2 i}\left(2 n b_{D}\right)\right\} .
\end{aligned}
$$

Then the system of integral equations takes the following form:

$$
\begin{gathered}
\sum_{p=1}^{2} \sum_{i=0}^{\infty} \sum_{j=0}^{\infty} \int_{0}^{t_{D}} c_{2 i j}^{p}(\tau) \Theta_{s 2 i}^{p}\left(\xi_{w}, \theta, t_{D}-\tau\right) \mathrm{Z}_{h j}\left(z_{D}, t_{D}-\tau\right) \mathrm{d} \tau=p_{D w u}\left(t_{D}\right) \\
\text { for }\left|z_{D}\right|<1 \text { and } \theta \in\left[0, b_{D}\right] \bigcup\left[\pi / 2-b_{D}, \pi / 2\right] \\
\qquad c_{00}^{1}\left(t_{D}\right)+c_{00}^{2}\left(t_{D}\right)=\frac{1}{2} .
\end{gathered}
$$

\section{Results}

First, we compare our solution with the vertically slotted-liner solution given by Spivak and Horne (1983), shown in Fig. 4, where the slots are parallel to each other in the $x$ direction. The Spivak and Horne (1983) dimensionless pressure values at $r_{D}=1$ were obtained from their Fig. 6 by digitizing the plot. As can be seen from Fig. 4, the Spivak and Horne (1983) solution is somewhat inaccurate, particularly at early times.

Figure 5 presents the comparison of our solutions with the solutions given by Onur (2012), Biryukov and Kuchuk (2012), and Hegeman (2012). For this case, an infinite reservoir vertically bounded from the top and bottom is used to generate the wellbore pressure data with the input model parameters that are given in Table 1. Our dimensionless pressure values were obtained by solving the system of integral equations given by Eqs. 82 and 83 . The difference between our analytical and the Hegeman (2012) numerical solutions for a 4-slot packer module is about $3 \%$, which is remarkably good. The difference between the Biryukov and Kuchuk (2012) uniform pressure solution for the partially penetrated packer with a totally open area and our solution for a 4 -slot packer module is about $12 \%$. It should also be noticed 


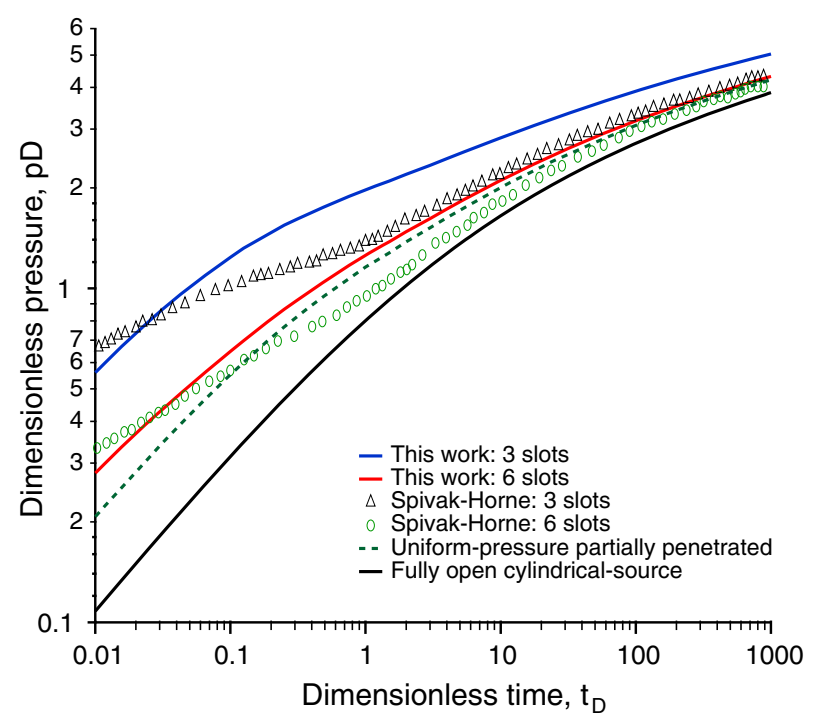

Fig. 4 Comparison of dimensionless pressures obtained from Spivak and Horne (1983) and our solutions for 3- and 6-slot cases

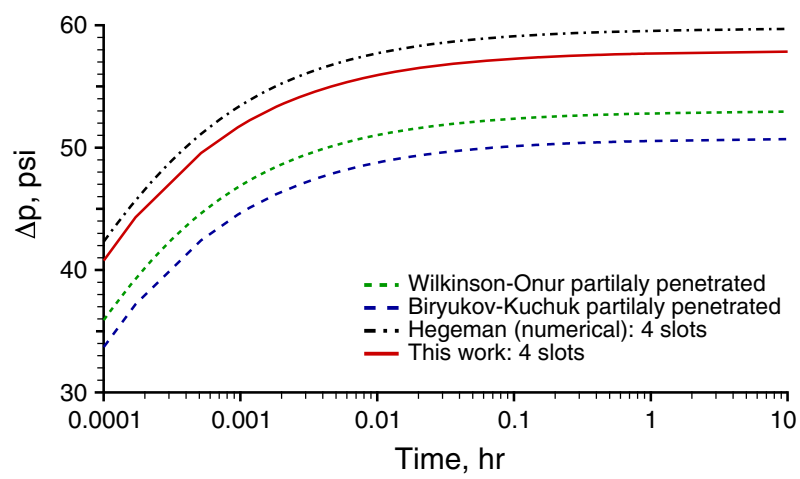

Fig. 5 Comparison of pressures changes obtained from Onur (2012) and Biryukov and Kuchuk (2012) for partially penetrated packer solutions, and Hegeman (2012) and our solutions for a 4-slot packer module

that there is about a $4 \%$ difference between the Onur (2012) and Biryukov and Kuchuk (2012) solutions due to the treatment of the uniform pressure condition over the open interval.

In Fig. 6, we present the comparison of the dimensionless uniform ( $p_{D w u}$ from Eqs. 82 and 83 ) and averaged ( $p_{D w a}$ from Eq. 51) pressures of a 4-slot packer module with a wellbore radius $\left(r_{w}\right)$ of $0.396 \mathrm{ft}$ for various $k_{v} / k_{h}$ values as a function of dimensionless time. As can be observed from this figure, the differences between two pressures are small (less than $12 \%)$ and change with time. For large times, the difference becomes a geometrical steadystate skin (does not change as a function of dimensionless time) when the system reaches spherical flow regime. This can be seen clearly in Fig. 7, which compares the derivatives of the uniform-and averaged-pressure solutions, when the derivatives exhibit a -1/2-slope spherical flow regimes, their derivatives become identical. For small times, the difference 
approaches zero because both the uniform pressure and averaged-pressure solutions become the same $\left(t_{D} \rightarrow 0 \quad p_{D}=\sqrt{\pi t_{D}}\right)$ and exhibit identical linear flow regimes.

Figure 8 presents derivatives for various anisotropy ratios, $k_{v} / k_{h}=0.01,0.1,1,10$, and 100 , for a 4-slot packer module in a vertically bounded reservoir, where the wellbore radius is $\left(r_{w}=0.396 \mathrm{ft}\right)$. For every anisotropy ratio case, the derivative clearly exhibits a $1 / 2$-slope linear flow regime at very early times. As we explain in the previous paragraph, the flow becomes linear in any flow systems that is internally bounded by a regular surface (cylinder, ellipse, line, sphere, etc.) as $t_{D} \rightarrow 0$ [see Page 48 of Kucuk (1978)].

As shown in Fig. 8, all derivatives go through a maximum after which they exhibit -1/2slope spherical flow regimes, which yields the spherical permeability $k_{s}=\left(k_{h}^{2} k_{v}\right)^{1 / 3}$. After the spherical flow regimes, the derivatives lead up to the radial flow regime, which yields the horizontal permeability $k_{h}$. The start and end of the spherical flow regimes, and the start of the radial flow regime are quite different for various $k_{v} / k_{h}$ values. As can be seen from Fig. 8 , the duration of the spherical flow regime of the $k_{v} / k_{h}=100$ case is very short, because due to a very high vertical permeability compared to the horizontal permeability, the effect of the no-flow top and bottom boundaries is observed at early times.

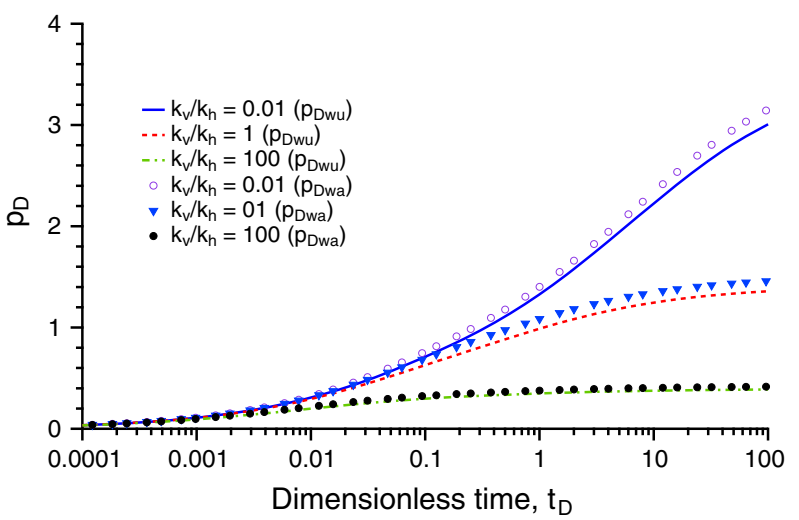

Fig. 6 Uniform $\left(p_{D w u}\right)$ and averaged $\left(p_{D w a}\right)$ dimensionless pressures of a 4-slot packer module with a wellbore radius $\left(r_{w}\right)$ of $0.396 \mathrm{ft}$ for various $k_{v} / k_{h}$ values

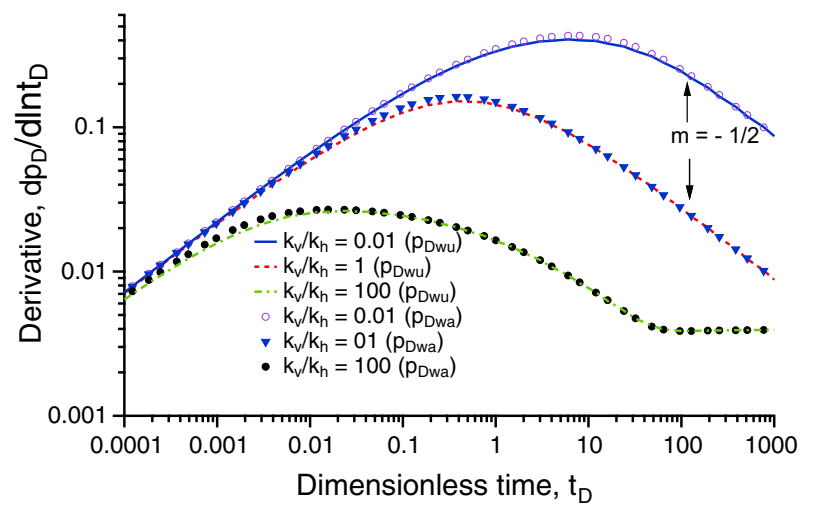

Fig. 7 Comparison of derivatives of uniform- and averaged-pressures of a 4-slot packer module with a wellbore radius $\left(r_{w}\right)$ of $0.396 \mathrm{ft}$ for various $k_{v} / k_{h}$ values 


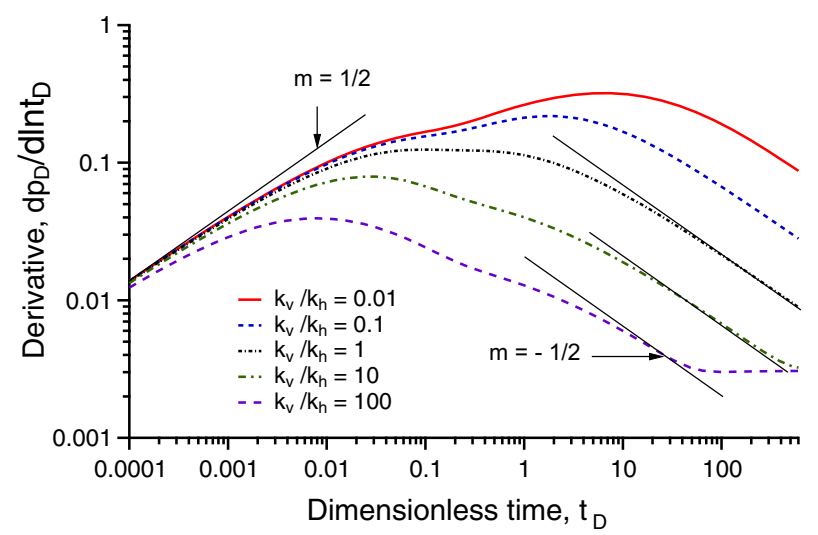

Fig. 8 Derivatives for a 4-slot packer module in a vertically bounded reservoir with wellbore radius $\left(r_{w}\right)$ of $0.396 \mathrm{ft}$ for various $k_{v} / k_{h}$ values

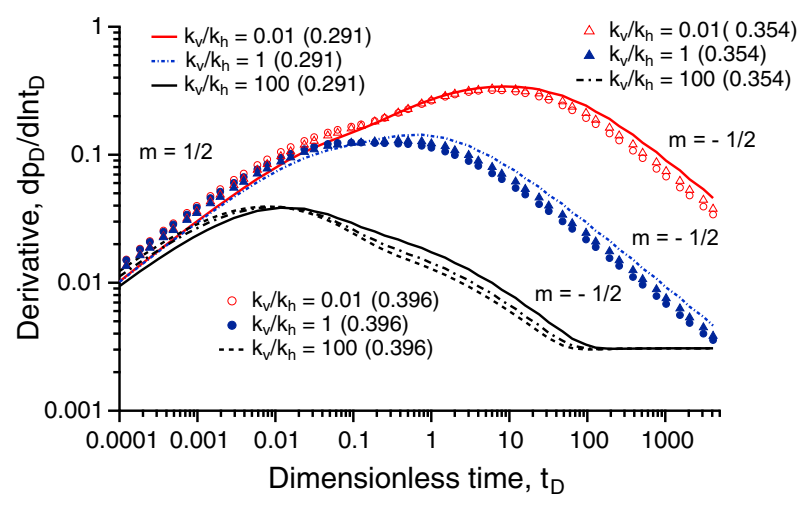

Fig. 9 Comparison of derivatives for a 4-slot packer module in a vertically bounded reservoir for various wellbore radii and $k_{v} / k_{h}$ values

Figure 9 compares the derivatives for a 4-slot packer module in a vertically bounded reservoir for various wellbore radii and $k_{v} / k_{h}$ values. The derivatives are almost the same at early times and slightly different during spherical flow regimes, where the effect of the wellbore radius becomes a steady-state pressure drop (geometrical skin) at late times.

\subsection{Drawdown Mobility}

During pretests or normal drawdown tests, the stabilized (spherical steady-state) drawdown pressure drop from the formation pressure can be considered as an indicator of a formation mobility. Under some simple assumptions regarding pretest, it is possible to relate the drawdown pressure to the formation mobility. Of course, if we know the skin factor then the drawdown formation mobility becomes an estimate, rather than an indicator, provided that there is no gas evolution around the slotted-packer.

For the 4-slot packer module, it is difficult to obtain a simple expression for the steady-state solution from the solutions given above. However, we can fit the dimensionless steady-state pressure to a second degree polynomial equation $\left(a+b x+c x^{2}\right)$ as a function of the natural 
logarithm of $k_{v} / k_{h}$ values for a given wellbore radius, as shown Fig. 10. For this plot, $r_{w}=0.396 \mathrm{ft}$. Thus, the dimensionless state-state pressure can be written as

$$
p_{D s s}=a+b \ln \left(\frac{k_{v}}{k_{h}}\right)+c\left[\ln \left(\frac{k_{v}}{k_{h}}\right)\right]^{2} .
$$

Table 2 presents the polynomial coefficients of Eq. 135 for the 4 -slot packer module for various wellbore radii. Equation 135 is an excellent fit for the dimensionless state-state pressure, as shown Fig. 10; the absolute relative error between the actual dimensionless pressure and the estimated one from Eq. 135 is less then $0.5 \%$ for $k_{v} / k_{h} \leq 1$ (i.e., horizontal permeability is greater than the vertical one) and less then $3 \%$ for $k_{v} / k_{h}>1$ (i.e., vertical permeability is greater than the horizontal one). It is well know that for most formations, the horizontal permeability is greater than the vertical permeability.

Using the definition of the wellbore dimensionless pressure given by Eq. 12 and the dimensionless steady-state pressure given by Eq. 135, the drawdown horizontal mobility $\left(k_{h} / \mu\right)$ for anisotropic media can be written as

$$
k_{h} / \mu=\frac{q p_{D s s}}{4 \pi l_{w} \Delta p_{w}}
$$

where $p_{D s s}$ is given by Eq. 135 , the steady-state pressure is defined as $\Delta p_{w}=p_{o}-p_{w}, p_{o}$ is the initial formation pressure measured by the slotted-packer module before the drawdown period or obtained from the buildup test, $q$ is the flow rate may vary between 0.1 and $50 \mathrm{~cm}^{3} / \mathrm{s}$ depending on formation parameters. To obtain $k_{h} / \mu$ from Eq. 136 we have to assume a reasonable $k_{v} / k_{h}$ value or is combined with the buildup spherical permeability.

A typical pretest sequence is: a drawdown test followed by a buildup test. During the drawdown test, mostly mud filtrate perhaps with a little volume of oil is produced from the

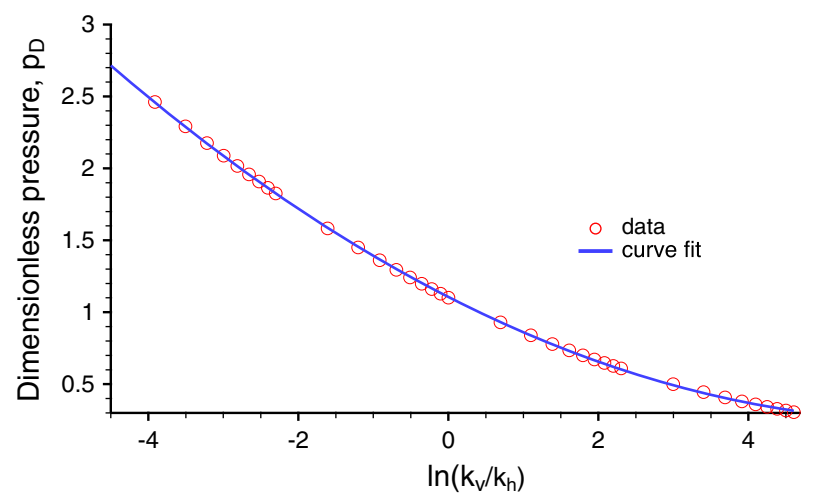

Fig. 10 Dimensionless pressure as a function of the natural logarithm of values of $k_{v} / k_{h}$ for a wellbore radius $\left(r_{w}\right)$ of $0.396 \mathrm{ft}$

Table 2 The polynomial coefficients of Eq. 135 for the 4-slot packer for various wellbore radii

\begin{tabular}{llll}
\hline$r_{w}$ & $a$ & $b$ & $c$ \\
\hline 0.291 & 1.1627 & -0.28288 & 0.021493 \\
0.328 & 1.1380 & -0.27592 & 0.021126 \\
0.354 & 1.1243 & -0.27168 & 0.020852 \\
0.396 & 1.1065 & -0.26574 & 0.020402 \\
\hline
\end{tabular}


4-slot packer module, and the produced volume is normally small. The pressure at the packer interval reaches quickly a spherical steady-state condition, unless the permeability formation is very low.

\section{Conclusions}

In this work, we have considered mixed boundary value problems for pressure transient diffusion in porous media arising in connection with the pressure transient behavior of formation tests conducted with the Wireline Formation Testing (WFT) vertically slotted-packer configuration in a vertical well. The solutions in this paper are essentially based on the idea of introducing an unknown flux density function on the open section (slot) of the wellbore, and its expansion in terms of singular basis functions. These functions reduce the mixed boundary value problem to a system of linear algebraic equations of modest size, which is easy to solve numerically. Such solutions also prove to be accurate, numerically stable. It is shown that the existing analytical solution is somewhat inaccurate. The anisotropy ratio $\left(k_{v} / k_{h}\right)$ significantly affects the pressure transient behavior of the slot packer module. The wellbore radius also affects the pressure transient behavior the system. Finally, we have given a formula to obtain the drawdown horizontal mobility $\left(k_{h} / \mu\right)$ for anisotropic media.

Acknowledgments The authors are grateful to Schlumberger for permission to publish this paper.

Open Access This article is distributed under the terms of the Creative Commons Attribution License which permits any use, distribution, and reproduction in any medium, provided the original author(s) and the source are credited.

\section{Appendix 1}

In this section we present an efficient way to evaluate the functions $Z_{j}, Z_{j h}$ and $\Theta_{2 i} . Z_{j}$ can be easily evaluated by passing into Laplace domain

$$
\begin{aligned}
\mathrm{Z}_{j}\left(z_{D}, u, t_{D}\right)= & \frac{2}{\sqrt{\pi t_{D}} v} \int_{0}^{\pi} e^{-\left(z_{D}-\cos \eta\right)^{2} /\left(4 v^{2} t_{D}\right)} \cos (j \eta) \mathrm{d} \eta \\
= & \frac{2}{\sqrt{\pi t_{D}} v} \mathcal{L}^{-1}\left(\int_{0}^{\pi} \frac{\cos (j \eta) \mathrm{d} \eta}{s+\left(z_{D}-\cos \eta\right)^{2}}, \frac{1}{4 v^{2} t_{D}}\right) \\
= & \frac{2}{\sqrt{\pi t_{D}} \nu} \mathcal{L}^{-1}\left(\frac{1}{2 i \sqrt{s}} \int_{0}^{\pi} \frac{\cos (j \eta) \mathrm{d} \eta}{\left(z_{D}+i \sqrt{s}-\cos \eta\right)}\right. \\
& \left.-\frac{\cos (j \eta) \mathrm{d} \eta}{\left(z_{D}-i \sqrt{s}-\cos \eta\right)}, \frac{1}{4 v^{2} t_{D}}\right) \\
= & \frac{2}{\sqrt{\pi t_{D}} v} \operatorname{Im} \mathcal{L}^{-1}\left(\frac{1}{\sqrt{s}} \int_{0}^{\pi} \frac{\cos (j \eta) \mathrm{d} \eta}{\left(z_{D}+i \sqrt{s}-\cos \eta\right)}, \frac{1}{4 v^{2} t_{D}}\right) .
\end{aligned}
$$

The last integral can be evaluated using the following result from Prudnikov et al. (1981)

$$
\int_{0}^{\pi} \frac{\cos (j \eta) \mathrm{d} \eta}{a+\cos \eta}= \begin{cases}\frac{\pi}{\sqrt{1-a^{2}}}\left(\sqrt{1-a^{2}}-a\right)^{j} & \text { if }|a| \leq 1 \\ \frac{\pi}{\sqrt{a^{2}-1}}\left(\sqrt{a^{2}-1}-a\right)^{j} & \text { if }|a|>1 .\end{cases}
$$


In the same way, the Laplace transform is useful when evaluating $Z_{j h}$. The Laplace transform of $Z_{h}$ can be written as follows (see Erdelyi (1954))

$$
\begin{aligned}
L\left[\mathrm{Z}_{h}\left(z_{D}, u, t_{D}\right)\right]= & \sum_{l=0}^{\infty} \frac{(-1)^{l} e^{-\left(z_{D}-u+2 z_{D w}+2 l h_{D}\right) \sqrt{s / v^{2}}}}{\sqrt{s} v}+\sum_{l=1}^{\infty}\left[\frac{e^{-\left(2 l h_{D}+u-z_{D}\right) \sqrt{s / v^{2}}}}{\sqrt{s} v}\right. \\
& \left.+\frac{e^{-\left(z_{D}-u+2 l h_{D}\right) \sqrt{s / v^{2}}}}{\sqrt{s} v}+\frac{(-1)^{l} e^{-\left(2 l h_{D}-z_{D}+u-2 z_{D w}\right) \sqrt{s / v^{2}}}}{\sqrt{s} v}\right] .
\end{aligned}
$$

The infinite summation can now easily be performed, we then obtain

$$
\begin{aligned}
L\left[\mathrm{Z}_{h}\left(z_{D}, u, t_{D}\right)\right]= & \frac{e^{-\left(z_{D}-u+2 z_{D w}\right) \sqrt{s / v^{2}}}}{\sqrt{s} v\left(1+e^{-2 h_{D} \sqrt{s / v^{2}}}\right)}+\frac{e^{-\left(2 h_{D}+u-z_{D}\right) \sqrt{s / v^{2}}}}{\sqrt{s} v\left(1-e^{-2 h_{D} \sqrt{s / v^{2}}}\right)} \\
& +\frac{e^{-\left(z_{D}-u+2 h_{D}\right) \sqrt{s / v^{2}}}}{\sqrt{s} v\left(1-e^{-2 h_{D} \sqrt{s / v^{2}}}\right)}+\frac{e^{-\left(2 h_{D}-z_{D}+u-2 z_{D}\right)} \sqrt{s / v^{2}}}{\sqrt{s} v\left(1+e^{-2 h_{D} \sqrt{s / v^{2}}}\right)},
\end{aligned}
$$

and consequently we get the following expression for the Laplace transform of $Z_{h j}$

$$
\begin{aligned}
L\left[\mathrm{Z}_{h j}\left(z_{D}, t_{D}\right)\right]= & \frac{\pi e^{-\left(z_{D}+2 z_{D w}\right) \sqrt{s / v^{2}}(-1)^{j} \mathrm{I}_{j}\left(\sqrt{s / v^{2}}\right)}+\frac{\pi e^{-\left(2 h_{D}-z_{D}\right) \sqrt{s / v^{2}}}(-1)^{j} \mathrm{I}_{j}\left(\sqrt{s / v^{2}}\right)}{2 \sqrt{2 s}\left(1-e^{-2 h_{D} \sqrt{s / v^{2}}}\right)}}{2 \sqrt{2 s}\left(1-e^{-2 h_{D} \sqrt{s / v^{2}}}\right)} \\
& +\frac{\pi e^{-\left(z_{D}+2 h_{D}\right) \sqrt{s / v^{2}}} \mathrm{I}_{j}\left(\sqrt{s / v^{2}}\right)}{2 \sqrt{2 s}\left(1-e^{-2 h_{D} \sqrt{s / v^{2}}}\right)}+\frac{\pi e^{-\left(2 h_{D}-z_{D}-2 z_{D w}\right) \sqrt{s / v^{2}}} \mathrm{I}_{j}\left(\sqrt{s / v^{2}}\right)}{2 \sqrt{2 s}\left(1-e^{-2 h_{D} \sqrt{s / v^{2}}}\right)},
\end{aligned}
$$

where $\mathrm{I}_{j}$ is the Modified Bessel function of the First kind.

Now let us consider the function $\Theta_{2 i}\left(1, \theta, t_{D}\right)$. It can be seen that the series terms in the Laplace domain in Eq. 34 are slowly convergent for $r_{D}=1$, but from Abramowitz and Stegun (1972) we can find that

$$
\frac{2 \mathrm{~K}_{\mathrm{n}}(\sqrt{s})}{\mathrm{K}_{\mathrm{n}-1}(\sqrt{s})+\mathrm{K}_{\mathrm{n}+1}(\sqrt{s})}=-\frac{\mathrm{K}_{\mathrm{n}}(\sqrt{s})}{\mathrm{K}_{\mathrm{n}}^{\prime}(\sqrt{s})} \sim \frac{\sqrt{s}}{\sqrt{s+n^{2}}}
$$

for large $n$. Thus, we can rewrite Eq. 34 for $r_{D}=1$ as

$$
\begin{aligned}
\Theta_{2 i}\left(1, \theta, t_{D}\right)= & \mathcal{L}^{-1}\left\{\frac{1}{\sqrt{s}} \sum_{n=0}\left(1-\frac{\delta_{0 n}}{2}\right)\left[\frac{2 \mathrm{~K}_{n}(\sqrt{s}) \mathbf{J}_{2 i}\left(n b_{D}\right) \cos (n \theta)}{\mathrm{K}_{n+1}(\sqrt{s})+\mathrm{K}_{n-1}(\sqrt{s})}-\frac{\sqrt{s} \cos n \theta}{\sqrt{s+n^{2}}}\right]\right\} \\
& +\frac{1}{\sqrt{\pi t_{D}}} \sum_{n=-\infty}^{\infty} e^{-n^{2} t_{D}} \mathbf{J}_{2 i}\left(n b_{D}\right) \cos (n \theta) .
\end{aligned}
$$

Now the first sum converges very fast (about 50 terms is enough to obtain 4-5 digits precision), while the second has a very high convergence rate for $t_{D} \geq 1$, due to the presence of the exponential term. The convergence rate, however, slows down rapidly for $t_{D}<1$, we, 
therefore, propose to rewrite it in the following form:

$$
\begin{aligned}
& \sum_{n=-\infty}^{\infty} \frac{e^{-n^{2} t_{D}}}{\sqrt{\pi t_{D}}} \mathrm{~J}_{2 i}\left(n b_{D}\right) \cos (n \theta) \\
& \quad=(-1)^{i} \int_{0}^{\pi} \sum_{n=-\infty}^{\infty} \frac{e^{-n^{2} t_{D}}}{\pi \sqrt{\pi t_{D}}} \cos \left(n\left[b_{D} \cos \alpha+\theta\right]\right) \cos (2 i \alpha) \mathrm{d} \alpha \\
& \quad=\frac{(-1)^{i}}{\sqrt{\pi} t_{D}} \sum_{n=-\infty}^{\infty} \int_{0}^{\pi} e^{-\frac{\left(2 \pi n / b_{D}+\theta / b_{D}+\cos \alpha\right)^{2}}{{ }^{4 t} / b_{D}^{2}}} \cos (2 i \alpha) \mathrm{d} \alpha .
\end{aligned}
$$

Note that here we used the Poisson summation formula. The last integral and summation can be easily evaluated exactly by the same way as we described for $Z_{j}$ and $Z_{h j}$ above. A similar technique without any significant changes can be also applied to evaluation of the function $\Theta_{N_{0} 2 i}$. It can also be shown that for large $n$

$$
\begin{aligned}
F c_{n}\left(\xi_{w}, \theta, \frac{a^{2} s}{4}\right) & \rightarrow \frac{2 \sqrt{2} \mathrm{~K}_{\mathrm{n}}\left(\sqrt{\frac{s a^{2}}{2} \sinh 2 \xi_{w}}\right) \cos n \theta}{\sqrt{s a^{2} \sinh 2 \xi_{w}}\left[\mathrm{~K}_{\mathrm{n}-1}\left(\sqrt{\frac{s a^{2}}{2} \sinh 2 \xi_{w}}\right)+\mathrm{K}_{\mathrm{n}+1}\left(\sqrt{\frac{s a^{2}}{2} \sinh 2 \xi_{w}}\right)\right]} \\
& \rightarrow \frac{\cos n \theta}{\sqrt{\frac{s a^{2}}{2} \sinh 2 \xi_{w}+n^{2}}},
\end{aligned}
$$

and

$$
\begin{aligned}
F s_{n}\left(\xi_{w}, \theta, \frac{a^{2} s}{4}\right) & \rightarrow \frac{2 \sqrt{2} \mathrm{~K}_{\mathrm{n}}\left(\sqrt{\frac{s a^{2}}{2} \sinh 2 \xi_{w}}\right) \sin n \theta}{\sqrt{s a^{2} \sinh 2} \xi_{w}\left[\mathrm{~K}_{\mathrm{n}-1}\left(\sqrt{\frac{s a^{2}}{2} \sinh 2 \xi_{w}}\right)+\mathrm{K}_{\mathrm{n}+1}\left(\sqrt{\frac{s a^{2}}{2} \sinh 2 \xi_{w}}\right)\right]} \\
& \rightarrow \frac{\sin n \theta}{\sqrt{\frac{s a^{2}}{2} \sinh 2 \xi_{w}+n^{2}}} .
\end{aligned}
$$

Thus, the series in Eqs. 115-116 can be rewritten as

$$
\begin{aligned}
\Theta_{2 i}^{p}\left(\xi, \theta, t_{D}\right)= & \mathcal{L}^{-1}\left\{\sum _ { n = 0 } \left[F c_{n}\left(\xi, \theta, \frac{a^{2} s}{4}\right) \cos \left(n \theta_{p}\right)+F s_{n}\left(\xi, \theta, \frac{a^{2} s}{4}\right) \sin \left(n \theta_{p}\right)\right.\right. \\
& \left.\left.-\left(1-\frac{\delta_{0 n}}{2}\right) \frac{\cos \left[n\left(\theta-\theta_{p}\right)\right]}{\sqrt{\frac{s a^{2}}{2} \sinh 2 \xi_{w}+n^{2}}}\right] \times \mathrm{J}_{2 i}\left(n b_{D}\right)\right\} \\
& +\frac{\sqrt{2}}{\sqrt{a^{2} \pi \sinh 2 \xi_{w} t_{D}}} \sum_{n=-\infty}^{\infty} e^{-\frac{2 n^{2} t_{D}}{a^{2} \sinh 2 \xi_{w}}} \mathbf{J}_{2 i}\left(n b_{D}\right) \cos \left[n\left(\theta-\theta_{p}\right)\right], \\
\Theta_{2 i+1}^{p}\left(\xi, \theta, t_{D}\right)= & \mathcal{L}^{-1}\left\{\sum _ { n = 1 } \left[F s_{n}\left(\xi, \theta, \frac{a^{2} s}{4}\right) \cos \left(n \theta_{p}\right)-F c_{n}\left(\xi, \theta, \frac{a^{2} s}{4}\right) \sin \left(n \theta_{p}\right)\right.\right. \\
& \left.\left.-\frac{\sin \left[n\left(\theta-\theta_{p}\right)\right]}{\sqrt{\frac{s a^{2}}{2} \sinh 2 \xi_{w}+n^{2}}}\right] \times \mathbf{J}_{2 i+1}\left(n b_{D}\right)\right\}
\end{aligned}
$$




$$
+\frac{\sqrt{2}}{\sqrt{a^{2} \pi \sinh 2 \xi_{w} t_{D}}} \sum_{n=-\infty}^{\infty} e^{-\frac{2 n^{2} t_{D}}{a^{2} \sinh 2 \xi w}} \mathbf{J}_{2 i+1}\left(n b_{D}\right) \sin \left[n\left(\theta-\theta_{p}\right)\right] .
$$

The last series in Eq. 147 has the same form as in the horizontally isotropic case, while the one in Eq. 148 can be rewritten as

$$
\begin{aligned}
& \frac{\sqrt{2}}{\sqrt{a^{2} \pi \sinh 2 \xi_{w} t_{D}}} \sum_{n=-\infty}^{\infty} e^{-\frac{2 n^{2} t_{D}}{a^{2} \sinh 2 \xi_{w}}} \mathbf{J}_{2 i+1}\left(n b_{D}\right) \sin \left[n\left(\theta-\theta_{p}\right)\right] \\
& =(-1)^{i} \int_{0}^{\pi} \sum_{n=-\infty}^{\infty} \frac{\sqrt{2} e^{-\frac{2 n^{2} t_{D}}{a^{2} \sinh 2 \xi_{w}}}}{\pi \sqrt{a^{2} \pi \sinh 2 \xi_{w} t_{D}}} \cos \left(n\left[b_{D} \cos \alpha+\theta-\theta_{p}\right]\right) \cos [(2 i+1) \alpha] \mathrm{d} \alpha \\
& =\frac{(-1)^{i}}{\sqrt{\pi} t_{D}} \sum_{n=-\infty}^{\infty} \int_{0}^{\pi} e^{-\frac{\left(2 \pi n / b_{D}+\left[\theta-\theta_{p}\right] / b_{D}+\cos \alpha\right)^{2}}{{ }^{4 t} t_{D} /\left(a^{2} b_{D}^{2} \cosh \xi_{w} \sinh \xi_{w}\right)}} \cos [(2 i+1) \alpha] \mathrm{d} \alpha,
\end{aligned}
$$

which can be summed up in the same way as we proposed for $Z_{j}$ and $Z_{h j}$. The last point on which we want to focus is the computation of integrals in Eq. 40 . On every subinterval $\left[t_{1}, t_{2}\right]$ the functions $\Theta_{i}^{(p)}\left(1, \theta, t_{D}\right), Z_{j}\left(z_{D}, t_{D}\right)$ and $Z_{h j}\left(z_{D}, t_{D}\right)$ can be approximated as follows

$$
\begin{gathered}
\Theta_{i}^{(p)}\left(1, \theta, t_{D}\right)=\frac{A_{i}(\theta)}{t^{1 / 2}}+\frac{B_{j}(\theta)}{t_{D}^{[i / 2]+1}}, \\
Z_{j}\left(z_{D}, t_{D}\right)=C_{j}\left(z_{D}\right)+\frac{D_{j}\left(z_{D}\right)}{t_{D}^{[j / 2]+1 / 2}}, \\
Z_{h j}\left(z_{D}, t_{D}\right)=\left[\frac{E_{j}\left(z_{D}\right)}{t_{D}^{1 / 2}}+\frac{F_{j}\left(z_{D}\right)}{t_{D}^{[j / 2]}}\right] e^{-\frac{\min \left(2 z_{D w}+z_{D}-1,2 h_{D}-2 z_{D w}-1\right)^{2}}{4 v^{2} t_{D}}} .
\end{gathered}
$$

We suggest computing the coefficients by a simple 2-point interpolation. Now the integral given in Eq. 40 can be written as

$$
\int_{a_{1}}^{a_{2}} \tau^{-\alpha} e^{-\beta / \tau} \mathrm{d} \tau=\int_{1 / a_{2}}^{1 / a_{1}} u^{\alpha-2} e^{-\beta u} \mathrm{~d} u=\frac{e^{-\beta / a_{2}}}{a_{2}^{\alpha-1}} \int_{1}^{a_{2} / a_{1}} v^{\alpha-2} e^{-\frac{\beta}{a_{2}}(v-1)} \mathrm{d} v .
$$

Although the last integral can be expressed in terms of incomplete gamma functions, one can see that for a 4-step time scheme we proposed above, $a_{2} / a_{1}$ can take only the following values: $2,1.5$, and $4 / 3$. Thus, this integral is a function of only one variable $\frac{\beta}{a_{2}}$ (while $a_{2} / a_{1}$ and $\alpha$ constitute only a finite set of parameters) and, therefore, can easily be approximated in a wide range using, for example, cubic splines for moderate values of $\frac{\beta}{a_{2}}$ and some simple asymptotic approximations for very small and very big values.

\section{References}

Abramowitz, M., Stegun, I.: Handbook of Mathematical Functions. Dover, New York (1972)

Al-Otaibi, S., Bradford, C., Zeybek, M., Corre, P.-Y., Slapal, M., Ayan, C., Kristensen, M.: Oil-water delineation with a new formation tester module. In: Paper SPE 159641, SPE Annual Technical Conference and Exhibition, San Antonio, Texas, USA, 8-10 October (2012)

Besson, J.: Performance of slanted and horizontal wells on an anisotropic medium. In: Paper SPE-20965-MS, European Petroleum Conference, The Hague, the Netherlands, 21-24 October (1990) 
Biryukov, D., Kuchuk, F.J.: Pressure transient solutions to mixed boundary value problems for partially open wellbore geometries in porous media. J. Pet. Sci. Eng. 96-97, 162-175 (2012)

Brons, F., Marting, V.E.: The effect of restricted fluid entry on well productivity. J. Pet. Technol. 13(2), 172-174 (1961)

Erdelyi, A.E.: Tables of Integral Transforms, vol. 1. McGraw-Hill (Bateman project), New York (1954)

Flores de Dios, T., Aguilar, M.G., Herrera, R.P., Garcia, G., Peyret, E., Ramirez, E., Arias, A., Corre, P.-Y., Slapal, M., Ayan, C.: New wireline formation tester development makes sampling and pressure testing possible in extra-heavy oils in Mexico. In: Paper SPE 159868, SPE Annual Technical Conference and Exhibition, San Antonio, Texas, USA, 8-10 October (2012)

Goode, P., Wilkinson, D.: Inflow performance of partially open horizontal wells. J. Pet. Technol. 43 (1991)

Gringarten, A.C., Ramey, H.J.J.: An approximate infinite conductivity solution for a partially penetrating line-source well. Trans. AIME 259, 140-148 (1975)

Hantush, M.S.: Non-steady flow to a well partially penetrating an infinite leaky aquifer. Proc. lraqi Sci. Soc. 1, 10-19 (1957)

Hegeman, P.: Sotted-wellbore numerical solution. Personal communication. (2012)

Kuchuk, F.: Pressure behavior of MDT packer module and DST in crossflow-multilayer reservoirs. J. Pet. Sci. Eng. 11(2), 123-135 (1994)

Kuchuk, F.: Interval pressure transient testing with MDT packer-probe module in horizontal wells. SPE Reservoir Eval. Eng. 1(6), 509-518 (1998)

Kuchuk, F., Onur, M., Hollaender, F.: Pressure Transient Formation and Well Testing: Convolution, Deconvolution and Nonlinear Estimation. Elsevier, New York (2010)

Kuchuk, F.J., Onur, M.: Estimating permeability distribution from 3D interval pressure transient tests. J. Pet. Sci. Eng. 39, 5-27 (2003)

Kuchuk, F.J., Wilkinson, D.: Pressure behavior of commingled reservoirs. SPE Form. Eval. 6(1), 111-120 (1991)

Kucuk, F.: Transient flow in elliptical systems. PhD thesis, Stanford University, Stanford, CA (1978)

Larsen, L.: The pressure-transient behavior of vertical wells with multiple flow entries. Paper SPE 26480, SPE Annual Technical Conference and Exhibition, 3-6 October, Houston, Texas, (1993)

Muskat, M.: The Flow of Homogeneous Fluids Through Porous Media. J. W. Edwards Inc., Ann Arbor (1937)

Nisle, R.G.: The effect of partial penetration on pressure build-up in oil wells. Trans. AIME 213, 85-90 (1958)

Odeh, A.S.: Steady-state flow capacity of wells with limited entry to flow. SPE J. 8(1), 43-51 (1968)

Onur, M.: Partially pettrated well model. Personal communication (2012)

Onur, M., Hegeman, P., Gok, I., Kuchuk, F.: A novel analysis procedure for estimating thickness-independent horizontal and vertical permeabilities from pressure data at an observation probe acquired by packerprobe wireline formation testers. SPE Reservoir Eval. Eng. 14(4), 457-472 (2011)

Onur, M., Hegeman, P., Kuchuk, F.: Pressure-transient analysis of dual packer-probe wireline formation testers in slanted wells. In: Paper SPE 90250, SPE Annual Technical Conference and Exhibition, Houston, Texas, 26-29 September (2004a)

Onur, M., Hegeman, P., Kuchuk, F.J.: Pressure-pressure convolution analysis of multiprobe and packer-probe wireline formation tester data. SPE Reservoir Eval. Eng. 7(5), 351-364 (2004b)

Onur, M., Kuchuk, F. J.: Integrated nonlinear regression analysis of multiprobe wireline formation tester packer and probe pressures and flow rate measurements. In: Paper SPE 56616, SPE Annual Technical Conference and Exhibition, Houston, 3-6 October (1999)

Ozkan, E., Raghavan, R.: New solutions for well-test-analysis problems: Part 1-Analytical considerations. SPE Form. Eval. 6(3), 359-368 (1991)

Papatzacos, P.: Approximate partial-penetration pseudoskin for infinite-conductivity wells. SPE Reservoir Eng. 2(2), 227-234 (1987a)

Papatzacos, P.: Exact solutions for infinite-conductivity wells. SPE Reservoir Eng. 2(2), 217-226 (1987b)

Pop, J., Badry, R., Morris, C., Wilkinson, D., Tottrup, P., Jonas, J.: Vertical interference testing with a wirelineconveyed straddle-packer tool. In: Paper SPE 26481, SPE Annual Technical Conference and Exhibition, Houston, Texas, 3-6 October (1993)

Prudnikov, A., Brychkov, Y., Marichev, O.I.: Integrali i ryadi. Nauka, Moscow, Elementarnie funkcii (in Russian) (1981)

Spivak, D., Horne, R.N.: Unsteady-state pressure response due to production with a slotted liner completion. J. Pet. Technol. 35(7), 1366-1372 (1983)

Streltsova-Adams, T.D.: Pressure drawdown in a well with limited flow entry. J. Pet. Technol. 31(11), 14691476 (1979)

Tang, Y., Ozkan, E., Kelkar, M., Sarica, C., Yildiz, T.: Performance of horizontal wells completed with slotted liners and perforations. In: Paper SPE 65516, SPE/CIM International Conference on Horizontal Well Technology, Calgary. Alberta, Canada, 6-8 (2000) 
Wilkinson, D., Hammond, P.: A perturbation method for mixed boundary-value problems in pressure transient testing. Trans. Porous Media 5, 609-636 (1990)

Schlumberger: Wireline-Schlumberger: Saturn 3D radial probe brochure (2012)

Yildiz, T.: Productivity of horizontal wells completed with screens. In: Paper SPE 76712, SPE Western Regional/AAPG Pacific Section Joint Meeting, Anchorage, Alaska, 20-22 May (2002a)

Yildiz, T.: Productivity of selectively perforated vertical wells. SPE J. 7(2), 158-169 (2002b)

Yildiz, T.: Productivity of horizontal wells completed with screens. Reservoir Eval. Eng. 7(5), 342-350 (2004)

Yildiz, T.: Assessment of total skin factor in perforated wells. Reservoir Eval. Eng. 9(1), 61-76 (2006a)

Yildiz, T.: Productivity of selectively perforated horizontal wells. SPE Prod. Oper. 21(1), 75-80 (2006b)

Yildiz, T., Bassiouni, Z.: Transient pressure analysis in partially-penetrating wells. In: Paper SPE-21551-MS, CIM/SPE International Technical Meeting, Calgary, Alberta, Canada, 10-13 June (1990)

Yildiz, T., Cinar, Y.: Inflow performance and transient pressure behavior of selectively completed vertical wells. SPE Reservoir Eval. Eng. 1(5), 467-475 (1998)

Zeybek, M., Kuchuk, F., Hafez, H.: Fault and fracture characterization using 3D interval pressure transient tests. In: Paper SPE 78506, Abu Dhabi International Petroleum Exhibition and Conference, Abu Dhabi, UAE, 13-16 October (2002)

Zimmerman, T., MacInnis, J., Hoppe, J., Pop, J., Long, T.: Application of emerging wireline formation testing technologies. In: OSEA 90105, 8th Offshore South East Asia Conference, Singapore, 4-7 December (1990) 